\title{
An improved holographic nodal line semimetal
}

\author{
Yan Liu and Xin-Meng Wu \\ Center for Gravitational Physics, Department of Space Science and \\ International Research Institute of Multidisciplinary Science, Beihang University, \\ Beijing 100191, China \\ E-mail: yanliu@buaa.edu.cn, wu_xm@buaa.edu.cn
}

\begin{abstract}
We study an improved holographic model for the strongly coupled nodal line semimetal which satisfies the duality relation between the rank two tensor operators $\bar{\psi} \gamma^{\mu \nu} \psi$ and $\bar{\psi} \gamma^{\mu \nu} \gamma^{5} \psi$. We introduce a Chern-Simons term and a mass term in the bulk for a complex two form field which is dual to the above tensor operators and the duality relation is automatically satisfied from holography. We find that there exists a quantum phase transition from a topological nodal line semimetal phase to a trivial phase. In the topological phase, there exist multiple nodal lines in the fermionic spectrum which are topologically nontrivial. The bulk geometries are different from the previous model without the duality constraint, while the resulting properties are qualitatively similar to those in that model. This improved model provides a more natural ground to analyze transports or other properties of strongly coupled nodal line semimetals.
\end{abstract}

KEYWORDS: Gauge-gravity correspondence, Holography and condensed matter physics (AdS/CMT)

ArXiv EPrint: 2012.12602 


\section{Contents}

1 Introduction 1

2 An improved holographic nodal line semimetal 3

2.1 Field model 3

2.1.1 From weakly coupled model to strongly coupled model 4

2.2 Holographic model 5

$\begin{array}{lll}2.2 .1 & \text { Zero temperature solutions }\end{array}$

3 ARPES from probe fermion $\quad 9$

$\begin{array}{llr}3.1 \text { Holographic fermionic spectral function } & 10\end{array}$

$\begin{array}{lll}3.2 & \text { Numerical results } & 13\end{array}$

4 Topological invariant in nodal line semimetal $\quad 16$

$\begin{array}{ll}4.1 \text { Topological invariant in field theory } & 16\end{array}$

$\begin{array}{lll}4.2 & \text { Topological invariant in holography } & 17\end{array}$

$\begin{array}{lll}5 & \text { Conclusion and discussion } & 19\end{array}$

$\begin{array}{lr}\text { A Equations of motion } & 20\end{array}$

$\begin{array}{ll}\text { B Counterterms and on-shell action } & 21\end{array}$

C Scaling symmetries and numerical calculation $\quad 22$

D Dirac system in the bulk $\quad 23$

D.1 Vielbein and spin connection 23

D.2 Gamma matrices and spinors 23

D.3 UV boundary terms of Dirac equations 24

D.4 IR boundary conditions 25

\section{Introduction}

Semimetals are critical states in the phase transition between insulators and conductors. In general, the Fermi surfaces in semimetals are of zero area and could be discrete points (Dirac semimetal or Weyl semimetal, i.e. DSM or WSM) or nodal lines (nodal line semimetal, i.e. NLSM). In topological semimetals these points or nodal lines are stable and could not be removed by perturbations of the system without breaking certain symmetries. ${ }^{1}$

\footnotetext{
${ }^{1}$ The nodal points or nodal lines in semimetals are the sources of the Berry curvature and give rise to topological charges [1, 2].
} 
Therefore topological semimetals exhibit lots of robust and exotic quantum properties and have attracted enormous research interests during the past few years $[2,3]$.

Topological semimetals are beyond the conventional Landau-Ginzburg paradigm and are characterized by the topological properties of the wave functions of the system. Most of the current models for topological semimetals are constructed based on the existence of quasiparticles, where one starts from a weakly coupled band theory of an effective Hamiltonian. An important and challenging question is whether and how the topological structures change if the system is strongly coupled. Without a clear quasiparticle description in the strongly coupled system [4], would nontrivial topological states still exist and if yes how could we describe them?

The holographic duality is a useful technique in describing strongly coupled systems in quantum physics by mapping them to simple gravitational problems [4-6]. Holography has been applied to the study of the topological nature in Weyl semimetals [7] and nodal line semimetals $[8,9]$ which uncovered lots of novel properties of topological semimetals at strong coupling [10-25]. See [26] for a recent review on this topic.

The holographic nodal line semimetals were first studied in $[8,9]$ and the key ingredients are a source for a rank two operator $\bar{\psi} \gamma^{\mu \nu} \psi$ and a mass deformation parameter. In [8, 9] a complex two form field is introduced in the bulk whose real part is dual to the above operator $\bar{\psi} \gamma^{\mu \nu} \psi$ while the imaginary part is dual to the other rank two operator $\bar{\psi} \gamma^{\mu \nu} \gamma^{5} \psi$. These two operators $\bar{\psi} \gamma^{\mu \nu} \psi$ and $\bar{\psi} \gamma^{\mu \nu} \gamma^{5} \psi$ on the field theory are not independent and we have the duality relation $\bar{\psi} \gamma^{\mu \nu} \gamma^{5} \psi=-\frac{i}{2} \varepsilon_{\alpha \beta}^{\mu \nu} \bar{\psi} \gamma^{\alpha \beta} \psi$. However, this duality relation has not been considered in the holographic model of $[8,9]$, where it is apparent that the two form field in the bulk is not exactly dual to the operators $\bar{\psi} \gamma^{\mu \nu} \psi$ and $\bar{\psi} \gamma^{\mu \nu} \gamma^{5} \psi$. In weakly coupled nodal line semimetals, the two operators $\bar{\psi} \gamma^{\mu \nu} \psi$ and $\bar{\psi} \gamma^{\mu \nu} \gamma^{5} \psi$ above are crucial to the related physics of nodal line semimetals. Therefore in holography it is more natural to take a two form field in the bulk to be exactly dual to these rank two operators in order to sharply describe the physics of strongly nodal line semimetals, as the holographic model in $[8,9]$ might include the physics of other rank two operators which is not related to the featured physics of nodal line semimetals. Thus we should consider an improved holographic nodal line semimetal model in which the duality relation of the rank two operators are indicated. ${ }^{2}$ A similar issue has been studied in the framework of AdS/QCD in [27] where a rank two field corresponds to vector mesons satisfying the self-dual constraint and it turns out that the bulk action should be a first order Chern-Simons term with a mass term. The duality of the operators on the boundary field theory automatically follows from the bulk equation of motion.

Our strategy is to follow the constructions in [27] to improve the holographic nodal line semimetal of $[8,9]$ to include the self-duality condition to make the holographic theory more natural. We shall focus on the zero temperature physics at which the topological properties are most manifest, by tuning the source of the rank two operators and the mass deformation. The bulk IR geometries vary with different emergent low energy symmetries. We also investigate the fermionic spectral function of this improved holographic model

\footnotetext{
${ }^{2}$ We thank Carlos Hoyos and Elias Kiritsis for helpful discussion on this point.
} 
and multiple nodal lines of the spectral function are found. The topological invariants of these nodal lines are also studied. From the field theoretical point of view a quantum phase transition is triggered by the strength of external sources, which is confirmed by studying the fermionic spectral functions. It is not obvious whether the duality constraint might induce any different effects on the holographic model, e.g. whether the order of the quantum phase transition might change, whether the topological state still exist and if yes then what are the corresponding topological invariants. We find that the properties in this improved holographic nodal line semimetal model share lots of similar physics as the one in $[8,9]$, i.e. there is no qualitative change. Therefore, our improved model serves as a natural holographic model for strongly coupled NLSM.

The organization of this paper is as follows. In section 2, we construct the improved holographic nodal line semimetal by including the self-duality condition. Zero temperature geometries of the system are studied to uncover the phase structures and the topological properties. In section 3 we study the fermionic spectral function to uncover the Fermi surface of the dual field theory. In section 4 we study the topological invariants of each nodal line of the fermionic spectal function. We conclude with discussions in section 5 . Some details of calculations are collected in four appendices.

\section{An improved holographic nodal line semimetal}

In this section we shall first analyze the field theory of nodal line semimetals and point out that we should consider the duality of rank two operators in the holographic construction. Then we follow [27] to improve the holographic nodal line semimetal of [8,9] and study the zero temperature solutions.

\section{$2.1 \quad$ Field model}

Topological nodal line semimetal is realized in a Lorentz violating field theoretical model [3, 8, 9, 28] with the following Lagrangian ${ }^{3}$

$$
\mathcal{L}=\bar{\psi}\left(\gamma^{\mu} \partial_{\mu}-m-\gamma^{\mu \nu} b_{\mu \nu}\right) \psi,
$$

where $\bar{\psi}=\psi^{\dagger} i \gamma^{0}, \gamma^{\mu \nu}=\frac{i}{2}\left[\gamma^{\mu}, \gamma^{\nu}\right]$ and $b_{\mu \nu}=-b_{\nu \mu}$ is an anti-symmetric two form field. If we turn on real $b_{x y}$, this system describes a nodal line semimetal. The equation of motion for the Dirac fermion is

$$
\left(\gamma^{\mu} \partial_{\mu}-m-\gamma^{\mu \nu} b_{\mu \nu}\right) \psi=0 .
$$

The Hamiltonian matrix can be obtained by writing this equation as a Schrodinger equation

$$
i \frac{\partial \psi}{\partial t}=-i \gamma^{0}\left(\gamma^{i} \partial_{i}-m-\gamma^{\mu \nu} b_{\mu \nu}\right) \psi \equiv \hat{H} \psi .
$$

Therefore, the band structure and the eigenstates of this Dirac system can be determined and reveal a quantum phase transition from the nodal line semimetal phase to gapped system by tuning the ratio between $b_{x y}$ and $m$.

\footnotetext{
${ }^{3}$ Note that we work in the Minkowski metric with most plus convention. The gamma matrices are the same as in appendix D.
} 

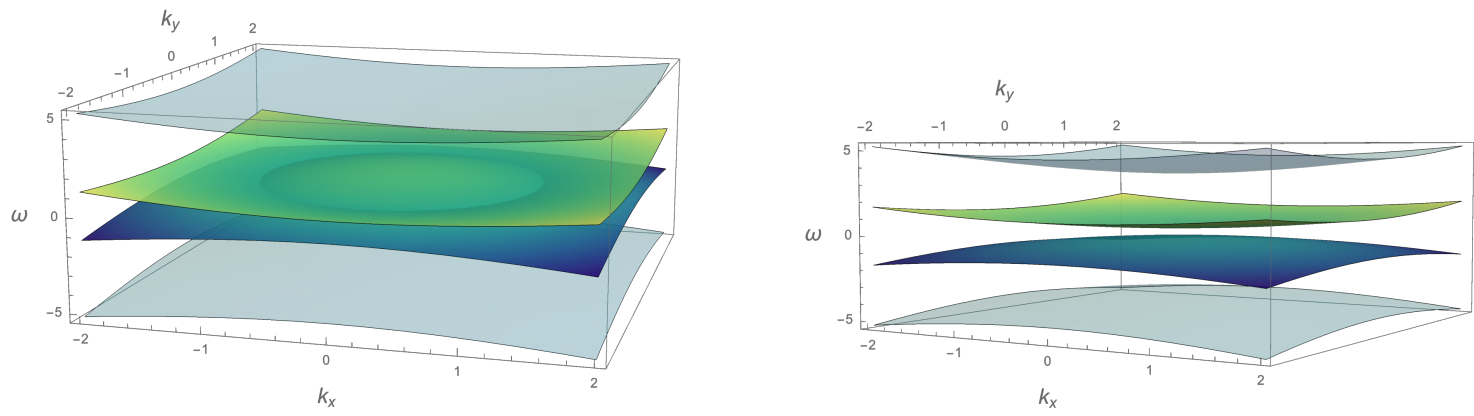

Figure 1. The illustration of the band structures with $k_{z}=0$ for the nodal line semimetal phase (left) and the gapped phase (right). In the nodal line semimetal phase, the crossing band forms a nodal circle where the excitations can be described by the Weyl fermions. There is no band crossing at any nonzero $k_{z}$.

However, in the four-dimensional Minkowski spacetime, the two anti-symmetric tensor operators $\bar{\psi} \gamma^{\mu \nu} \psi$ and $\bar{\psi} \gamma^{\mu \nu} \gamma^{5} \psi$ are not independent and are related by the duality relation

$$
\bar{\psi} \gamma^{\mu \nu} \gamma^{5} \psi=-\frac{i}{2} \varepsilon^{\mu \nu}{ }_{\alpha \beta} \bar{\psi} \gamma^{\alpha \beta} \psi
$$

where $\varepsilon_{t x y z}=1, \bar{\psi} \gamma^{\mu \nu} \psi$ is a pure real tensor operator while $\bar{\psi} \gamma^{\mu \nu} \gamma^{5} \psi$ is a pure imaginary tensor operator. As a consequence, it is more natural to take the operator $\bar{\psi} \gamma^{\mu \nu} \gamma^{5} \psi$ into consideration and the action (2.1) is modified into

$$
\mathcal{L}=\bar{\psi}\left(\gamma^{\mu} \partial_{\mu}-m-\gamma^{\mu \nu} b_{\mu \nu}+\gamma^{\mu \nu} \gamma^{5} b_{\mu \nu}^{5}\right) \psi
$$

Due to the duality relation of the two form operators (2.4), we turn on $b_{x y}, b_{y x}=-b_{x y}$ and their dual part of $b_{\mu \nu}^{5}$, i.e., $b_{t z}^{5}=-b_{z t}^{5}=i b_{x y}$. Here the external source $b_{\mu \nu}^{5}$ is set to be pure imaginary to make the Hamiltonian real. With this choice of Lagrangian in (2.5), the band structure, i.e. $E_{ \pm}= \pm \sqrt{\left(4 b_{x y} \pm \sqrt{m^{2}+k_{x}^{2}+k_{y}^{2}}\right)^{2}+k_{z}^{2}}$ is the same as that described with the Lagrangian of $(2.1)$ up to a prefactor of $b_{x y}[8,9]$. The band crossing appears at the $k_{z}=0$ plane and forms a circle with a radius $\sqrt{k_{x}^{2}+k_{y}^{2}}=\sqrt{16 b_{x y}^{2}-m^{2}}$ when $16 b_{x y}^{2}-m^{2}>0$, while for finite $k_{z}$ the energy band is gapped. The model (2.5) with the duality relation of the rank two operators (2.4) is called the improved nodal line semimetal. In this model, there still exists a quantum phase transition from a nodal line semimetal to a gapped phase, as shown in figure 1 .

\subsubsection{From weakly coupled model to strongly coupled model}

Starting from the properties of weakly coupled model for nodal line semimetal (2.5), we will construct a holographic model for strongly coupled nodal line semimetal with the duality relation. In holography, we use a complex anti-symmetric two form field $B_{\mu \nu}$ to be dual to operators $\bar{\psi} \gamma^{\mu \nu} \psi$ and $\bar{\psi} \gamma^{\mu \nu} \gamma^{5} \psi$. More precisely, we parameterize the complex $B_{\mu \nu}$ as $B_{\mu \nu}=$ $\frac{1}{\sqrt{2}}\left(B_{+\mu \nu}+i B_{-\mu \nu}\right)$ with the real anti-symmetric fields $B_{+\mu \nu}$ and $B_{-\mu \nu}$ the real and imaginary part in the $B_{\mu \nu}$ field, i.e. $B_{+\mu \nu}$ and $B_{-\mu \nu}$, duals to $\bar{\psi} \gamma^{\mu \nu} \psi$ and $\bar{\psi} \gamma^{\mu \nu} \gamma^{5} \psi$, respectively. 
The complex two form field $B_{a b}$ in the gravitational theory is dynamical. Note that $B_{a r}$ vanishes with a radial gauge. We expect the components $B_{\mu \nu}$ to be consistent with the duality condition between two tensor operators (2.4). Similar issue has been discussed in AdS/QCD and there are many progresses in imposing this self-duality relation in the gravitational theory. In particular, it has been proposed in $[27,29]$ that the action of the two form field $B_{a b}$ should be of first order, in such a way that the four-dimensional components satisfy a complex self-duality relation. The Chern-Simons term of $B_{a b}$ together with the mass term has been used in an improved holographic QCD model [27] to investigate the physical properties of mesons, from which the self-duality relation follows from the equation of motion directly instead of being imposed as particular boundary conditions. ${ }^{4}$ In this paper, we follow this approach to construct the improved holographic nodal line semimetal model that satisfies the self-duality condition.

\subsection{Holographic model}

The action of the improved holographic nodal line semimetal model is

$$
\begin{aligned}
S= & \int d^{5} x \sqrt{-g}\left[\frac{1}{2 \kappa^{2}}\left(R+\frac{12}{L^{2}}\right)-\frac{1}{4} \mathcal{F}^{2}-\frac{1}{4} F^{2}+\frac{\alpha}{3} \epsilon^{a b c d e} A_{a}\left(3 \mathcal{F}_{b c} \mathcal{F}_{d e}+F_{b c} F_{d e}\right)\right. \\
& \left.-\left(D_{a} \Phi\right)^{*}\left(D^{a} \Phi\right)-V_{1}(\Phi)-\frac{1}{6 \eta} \epsilon^{a b c d e}\left(i B_{a b} H_{c d e}^{*}-i B_{a b}^{*} H_{c d e}\right)-V_{2}\left(B_{a b}\right)-\lambda|\Phi|^{2} B_{a b}^{*} B^{a b}\right],
\end{aligned}
$$

where $\mathcal{F}_{a b}=\partial_{a} V_{b}-\partial_{b} V_{a}$ is the vector gauge field strength, $F_{a b}=\partial_{a} A_{b}-\partial_{b} A_{a}$ is the axial gauge field strength, $\epsilon^{a b c d e}$ is the upper indexed Levi-Civita tensor, and $D_{a}=\nabla_{a}-$ $i q_{1} A_{a}$ is the covariant derivative. ${ }^{5}$ Note that $\alpha$ is the Chern-Simons coupling. $B_{a b}$ is an antisymmetric complex two form field that duals to the two tensor operators $\bar{\psi} \gamma^{\mu \nu} \psi$ and $\bar{\psi} \gamma^{\mu \nu} \gamma^{5} \psi$, and

$$
H_{a b c}=\partial_{a} B_{b c}+\partial_{b} B_{c a}+\partial_{c} B_{a b}-i q_{2} A_{a} B_{b c}-i q_{2} A_{b} B_{c a}-i q_{2} A_{c} B_{a b}
$$

where $q_{2}$ is the axial charge of the two form field. $\eta$ is the coupling strength of the two form field. The potential terms are chosen as

$$
V_{1}=m_{1}^{2}|\Phi|^{2}+\frac{\lambda_{1}}{2}|\Phi|^{4}, \quad V_{2}=m_{2}^{2} B_{a b}^{*} B^{a b},
$$

where $m_{1}^{2}$ and $m_{2}^{2}$ are the mass parameters of the scalar field and the two form field. The $\lambda$ term denotes the interaction between the scalar field and the two form field. It should be noticed that in the previous holographic model [8,9] the self-duality of $B_{a b}$ is absent, where a canonical kinetic term together with the potential terms were used to describe the dynamics of $B_{a b}$. In this improved holographic nodal line semimetal model (2.6) we follow the approach in an AdS/QCD model [27] to use the Chern-Simons term and the mass term of $B_{a b}$ to describe the dynamics in the gravitational theory. Different from the AdS/QCD model [27] working in the probe limit around a pure $\mathrm{AdS}_{5}$ background,

\footnotetext{
${ }^{4} \mathrm{~A}$ kinetic term of $B_{a b}$ in the action is also allowed to be consistent with the self-duality condition and can be chosen to vanish without loss of generality.

${ }^{5}$ Note that $\epsilon_{a b c d e} \equiv \sqrt{-g} \varepsilon_{a b c d e}$, with $\varepsilon_{a b c d e}$ the Levi-Civita symbol and $\varepsilon_{0123 r}=1$.
} 
we concentrate on how this two form field together with a scalar field deforms the bulk geometry under continuously varying boundary sources. From the viewpoint of RG flow, this can be interpreted as a UV fixed point flows to an IR fixed point induced by the external sources. We will also study the properties of Fermi surfaces and topological structures of the dual filed theories.

After a variation of the total action with respect to the gauge fields, we can obtain the dual consistent currents and they satisfy

$$
\begin{aligned}
\partial_{\mu} J_{\text {con }}^{\mu}=0, & \\
\partial_{\mu} J_{5 \text { con }}^{\mu}=\lim _{r \rightarrow \infty} \sqrt{-g}( & -\frac{\alpha}{3} \epsilon^{r \alpha \beta \rho \sigma}\left(F_{\alpha \beta} F_{\rho \sigma}+\mathcal{F}_{\alpha \beta} \mathcal{F}_{\rho \sigma}\right)+i q_{1}\left[\Phi^{*}\left(D^{r} \Phi\right)-\Phi\left(D^{r} \Phi\right)^{*}\right] \\
& \left.-\frac{q_{2}}{\eta} \epsilon^{r \alpha \beta \rho \sigma} B_{\alpha \beta} B_{\rho \sigma}^{*}\right)+ \text { c.t. . }
\end{aligned}
$$

Here we have not explicitly shown the counterterm for simplicity and the above conservation can be further simplified in the radial gauge. The point is that the last two terms contribute only when the non-normalizable mode of the scalar filed or two form field is switched on and it is straightforward to see that the above identities are of the same structure of the weakly coupled theory. Thus this holographic model is expected to go beyond the weakly coupled theory to a strongly coupled nodal line semimetal model.

\subsubsection{Zero temperature solutions}

We focus on the ground state of this system and make the following ansatz for the zero temperature solution

$$
\begin{aligned}
d s^{2} & =u\left(-d t^{2}+d z^{2}\right)+\frac{d r^{2}}{u}+f\left(d x^{2}+d y^{2}\right), \\
\Phi & =\phi \\
B_{x y} & =-B_{y x}=\mathcal{B}_{x y} \\
B_{t z} & =-B_{z t}=i \mathcal{B}_{t z}
\end{aligned}
$$

in the coordinates $\{t, x, y, z, r\}$, where all the fields $u, f, \phi, \mathcal{B}_{x y}, \mathcal{B}_{t z}$ are real functions of the radial coordinate $r$. Note that the component $B_{x y}$ is real while $B_{t z}$ is pure imaginary. Substituting this ansatz into the equation of motion ${ }^{6}$ derived from the action (2.6), we write down the first order differential equations of $\mathcal{B}_{x y}$ and $\mathcal{B}_{t z}$

$$
\begin{aligned}
\mathcal{B}_{t z}^{\prime}-\frac{\eta \sqrt{u}}{2 f}\left(m_{2}^{2}+\lambda \phi^{2}\right) \mathcal{B}_{x y} & =0 \\
\mathcal{B}_{x y}^{\prime}-\frac{\eta f}{2 u^{\frac{3}{2}}}\left(m_{2}^{2}+\lambda \phi^{2}\right) \mathcal{B}_{t z} & =0 .
\end{aligned}
$$

We make some observations and explanations at this stage.

- $\mathcal{B}_{t z}$ and $\mathcal{B}_{x y}$ are two independent functions of $r$ and cannot be set to equal.

\footnotetext{
${ }^{6}$ The equations of motion for this system can be found in appendix A.
} 
- Close to UV $\mathrm{AdS}_{5}$ boundary $(r \rightarrow \infty), u \rightarrow r^{2}, f \rightarrow r^{2}$ while $\phi \rightarrow 0$. It is the term $\eta \cdot m_{2}^{2}$ that controls the conformal dimension of $B_{a b}$. In the following, we will fix $m_{1}^{2}=-3, m_{2}^{2}=1$ and $\eta=2$ to make the expected conformal dimensions for the operators which are dual to $\Phi$ and $B_{a b}$. For simplicity, we choose the couplings $q_{1}=q_{2}=1, \lambda=1$ and $\lambda_{1}=0.1$.

- With this choice of parameters, the leading order solutions of $\mathcal{B}_{t z}$ and $\mathcal{B}_{x y}$ are the same close to UV boundary, i.e. $\mathcal{B}_{t z}=\mathcal{B}_{x y} \simeq b r+\cdots$. This feature follows automatically from the dynamical equations of motion above and therefore the self-duality of the two form field is imposed in holography. Note that when the self-duality constraint is automatically imposed on the source terms, the duality condition should be also on the rank two tensor operators as long as we perform the variational principle on the dual field theory correctly. ${ }^{7}$

Therefore, we impose the following boundary condition which encode the duality relation of the rank two operators

$$
\lim _{r \rightarrow \infty} r \phi=M, \quad \lim _{r \rightarrow \infty} r^{-1} \mathcal{B}_{t z}=\lim _{r \rightarrow \infty} r^{-1} \mathcal{B}_{x y}=b,
$$

where $M$ plays the role of the source of the scalar operator $\bar{\psi} \psi$ while $b$ corresponds to the external source term of the tensor operators $\bar{\psi} \gamma^{\mu \nu} \psi$ and $\bar{\psi} \gamma^{\mu \nu} \gamma^{5} \psi$.

In the remaining context of this section, we illuminate the existence of three phases when tuning the dimensionless ratio between the external source strength of the scalar and tensor operators in field theory, i.e., $M / b$. From the numerical study of the free energy we found that the quantum phase transition is continuous, although the discontinuity appears in the IR-region of the bulk, i.e. $r \rightarrow 0$ in our choice of coordinates.

Topological nodal line semimetal $(M / b<\mathbf{0 . 8 5 9 7})$. The IR geometry of topological nodal line semimetal phase behaves as

$$
\begin{aligned}
u & =\frac{1}{8}(11+3 \sqrt{13}) r^{2}\left(1+\delta u r^{\alpha_{1}}\right), \\
f & =\sqrt{\frac{2}{3} \sqrt{13}-2} b_{x y 0} r^{\alpha}\left(1+\delta f r^{\alpha_{1}}\right), \\
\phi & =\phi_{0} r^{\beta} \\
\mathcal{B}_{t z} & =\frac{1}{8} \sqrt{54+15 \sqrt{13}} r^{2}\left(1+\delta b_{t z} r^{\alpha_{1}}\right), \\
\mathcal{B}_{x y} & =b_{x y 0} r^{\alpha}\left(1+\delta b_{x y} r^{\alpha_{1}}\right),
\end{aligned}
$$

where

$$
\left(\alpha, \beta, \alpha_{1}\right)=(0.183,0.228,1.273), \quad\left(\delta f, \delta b_{t z}, \delta b_{x y}\right)=(-2.616,1.720,-0.302) \delta u .
$$

In the IR limit $r \rightarrow 0, d s^{2}$ and $B_{a b} d x^{a} d x^{b}$ are invariant under the transformation $\left(r^{-1}, t, z\right) \rightarrow c\left(r^{-1}, t, z\right),(x, y) \rightarrow c^{\alpha / 2}(x, y)$. This implies that there is an emergent Lifshitztype symmetry in the deep IR region. We can set $b_{x y 0}=1$ using the scaling symmetry in

\footnotetext{
${ }^{7}$ We thank Carlos Hoyos for useful discussion on this point.
} 
the $x-y$ plane, i.e. the second type of scaling symmetry in appendix C. The above emergent Lifshitz scaling symmetry can be used to set $\delta u= \pm 1$. With $\delta u=-1$ the IR geometry flows to an asymptotic $\mathrm{AdS}_{5}$ boundary. Therefore we can take $\phi_{0}$ as the shooting parameter, which generates a class of solutions with a single dimensionless UV parameter $M / b$. By continuously varying $\phi_{0}$ in IR, the UV geometries are the AdS boundary with continuous $M / b$. We found that this type of IR geometry exist only when $M / b<0.8597$. The connection between this type of geometry and nodal line semimetal will be discussed in section 3 .

Quantum critical point $\left((M / b)_{c} \simeq \mathbf{0 . 8 5 9 7}\right)$. The IR geometry of the quantum critical point is

$$
\begin{aligned}
u & =u_{c} r^{2}\left(1+\delta u r^{\beta}\right), \\
f & =f_{c} r^{\alpha_{c}}\left(1+\delta f r^{\beta}\right), \\
\phi & =\phi_{c}\left(1+\delta \phi r^{\beta}\right), \\
\mathcal{B}_{t z} & =b_{t z c} r^{2}\left(1+\delta b_{t z} r^{\beta}\right), \\
\mathcal{B}_{x y} & =b_{x y c} r^{\alpha_{c}}\left(1+\delta b_{x y} r^{\beta}\right),
\end{aligned}
$$

with

$$
\left(u_{c}, f_{c}, \alpha_{c}, \phi_{c}, b_{t z c}\right)=\left(2.735,0.754 b_{x y c}, 0.314,0.557,1.437\right) \text {, }
$$

and

$$
\beta=1.274, \quad\left(\delta u, \delta f, \delta b_{t z}, \delta b_{x y}\right)=(0.882,-2.151,1.718,-0.254) \delta \phi .
$$

The Lifshitz type symmetry also emerges at the deep IR region, i.e., the geometry is invariant under the transformation $\left(r^{-1}, t, z\right) \rightarrow c\left(r^{-1}, t, z\right),(x, y) \rightarrow c^{\alpha_{c} / 2}(x, y)$ when $r \rightarrow 0$. Using this symmetry we can set $\delta \phi=-1$. We can also set $b_{x y c}=1$ using the scaling symmetry in the $x$-y plane. We obtain a unique geometry and in UV we have a special value of $M / b \simeq 0.8597$. An interesting observation is that, the critical value in this improved model is approximately half of that in the previous model $[8,9]$. Recall that in the field theoretical models, the improved model with $b_{x y}=1 / 2$ produces exactly the same band structure as the previous one in $[8,9]$ with $b_{x y}=1$. The critical value $(M / b)_{c}$ changes correspondingly with a factor $1 / 2$ in two models seems to also hold in holography, although the detailed geometries in the bulk are different.

Topological trivial phase $(M / b>\mathbf{0 . 8 5 9 7})$. The IR geometry for the trivial phase is

$$
\begin{aligned}
u & =\left(1+\frac{3}{8 \lambda_{1}}\right) r^{2}, \\
f & =r^{2} \\
\phi & =\sqrt{\frac{3}{\lambda_{1}}}+\phi_{1} r^{2\left(\sqrt{\frac{3+20 \lambda_{1}}{3+8 \lambda_{1}}}-1\right)}, \\
\mathcal{B}_{t z} & =\left(1+\frac{3}{8 \lambda_{1}}\right) b_{1} r^{2 \sqrt{2} \frac{3 \lambda+\lambda_{1}}{\sqrt{\lambda_{1}\left(3+8 \lambda_{1}\right)}}}, \\
\mathcal{B}_{x y} & =b_{1} r^{2 \sqrt{2} \frac{3 \lambda+\lambda_{1}}{\sqrt{\lambda_{1}\left(3+8 \lambda_{1}\right)}}} .
\end{aligned}
$$



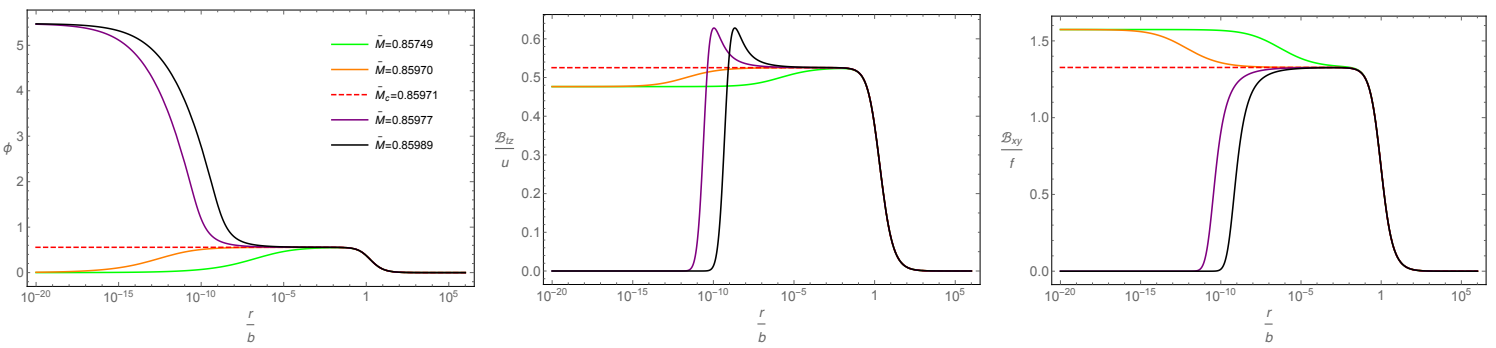

Figure 2. Bulk profiles for background fields as a function of the radial coordinate in three different phases in the vicinity of critical point. Different colors are for different values of $\hat{M}=M / b$.

We can set $b_{1}=1$ using the scaling symmetries and take $\phi_{1}$ as the shooting parameter. This type of IR geometry only exist for $M / b>0.8597$, when we continuously tune the shooting parameter $\phi_{1}$.

The profiles of $\phi, \mathcal{B}_{t z} / u$ and $\mathcal{B}_{x y} / f$ for parameters close to $(M / b)_{c}$ as a function of radial coordinate are illustrated in figure 2. As we gradually tune $M / b$ these profiles change smoothly from boundary to an intermediate scale $r / b \propto 10^{-5}$. However, the matter fields flow discontinuously to different types of IR profiles. Close to $(M / b)_{c}$, the geometry for three phases first flows to an intermediate Lifshitz type geometry from UV and then splits into different types of IR geometry.

We can compare the background geometries in the holographic models with and without the imposed the self-dual constraint. There is an extra dynamical component in $B_{a b}$ in the improved NLSM model. The profiles of $\phi$ and $B_{x y}$, as well as the type of the emergent symmetries at low-energy are similar to the model $[8,9]$ in all the three phases. Then we come to the question what the topological properties are in this improved NLSM model, which will be studied in the next sections where we studied the properties of fermionic operator by probing massive fermions in the bulk.

Finally, the dependence of the free energy density as a function of $M / b$ in this system is shown in figure 3 . We find that the free energy density smoothly across the quantum phase transition point as $M / b$ increase and the first derivative of the free energy density with respect to $M / b$ reaches the same value in the vicinity of $(M / b)_{c}$ from two phases. Therefore, we conclude the topological phase transition in this improved NLSM is a continuous phase transition, while the self-duality does not change the order of the transition.

\section{ARPES from probe fermion}

The remaining of this paper is to provide evidence of the topological band structure in the improved holographic nodal line semimetal and the existence of quantum phase transition. In experiments, angle resolved photoemission spectroscopy (ARPES) has been used to discover nodal band structure. Theoretically, this motivates us to investigate the lowenergy effective topological Hamiltonian in the holographic nodal line semimetal phase, which is defined from the zero frequency Green's function of the Dirac fermionic operators, i.e., $H_{t}(\vec{k})=-G^{-1}(0, \vec{k})[37,38]$. From the topological Hamiltonian one could obtain the topological properties of the nodal lines. 


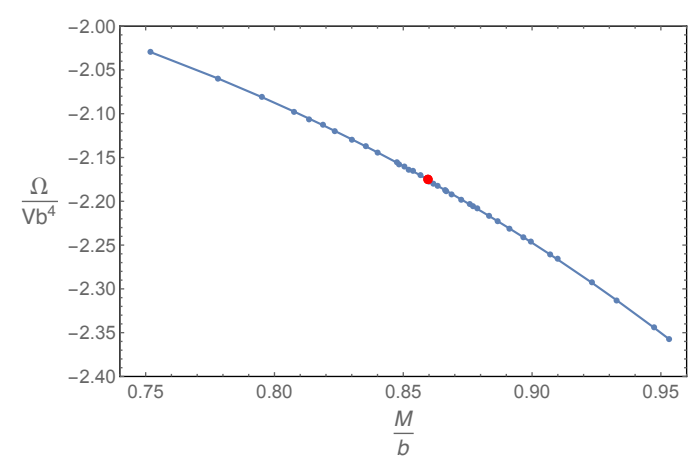

Figure 3. The free energy density as a function of $M / b$ across the critical point (the red dot). The free energy density is continuous and smooth during the topological quantum phase transition.

In the following we shall use the same strategy as $[8,9]$ to study the fermionic spectral function by probing a fermion in the bulk of our model. We shall compare the results of this improved model and the previous model without duality condition of the two form operators.

\subsection{Holographic fermionic spectral function}

In holography, we can obtain the fermionic spectral function by probing a single fermion in the gravitational bulk geometry [31, 32]. However, in the four dimensional boundary field theory with a five dimensional bulk dual, the Dirac fermion in the bulk corresponds to chiral fermionic operator in the field theory $[8,9,33,36]$. To obtain a Dirac fermionic operators in the boundary field theory with nontrivial spectral structure, in bulk we need to consider two sets of Dirac fermions coupled with each other through the scalar field $\Phi$ and the self-dual two form field $B_{a b}[8,9]$ with the following action

$$
\begin{aligned}
S_{\text {fermion }} & =S_{1}+S_{2}+S_{\mathrm{int}}+S_{\mathrm{bdy}} \\
S_{\mathrm{int}} & =S_{\Phi}+S_{B}
\end{aligned}
$$

where $S_{\text {bdy }}$ is the boundary term to make the theory self-consistent and

$$
\begin{aligned}
& S_{1}=\int d^{5} x \sqrt{-g} \bar{\Psi}_{1}\left(\Gamma^{a} D_{a}-m_{f}\right) \Psi_{1}, \\
& S_{2}=\int d^{5} x \sqrt{-g} \bar{\Psi}_{2}\left(\Gamma^{a} D_{a}+m_{f}\right) \Psi_{2}
\end{aligned}
$$

are the action for two types of free fermions with opposite signs of mass and with different quantizations where $\bar{\Psi}=\Psi^{\dagger} i \Gamma^{0}$ and $D_{a}=\nabla_{a}-i q_{3} A_{a}$, while

$$
\begin{aligned}
& S_{\Phi}=-\int d^{5} x \sqrt{-g}\left(\eta_{1} \Phi \bar{\Psi}_{1} \Psi_{2}+\eta_{1}^{*} \Phi^{*} \bar{\Psi}_{2} \Psi_{1}\right) \\
& S_{B}=\int d^{5} x \sqrt{-g}\left(\eta_{2} B_{a b} \bar{\Psi}_{1} \Gamma^{a b} \gamma^{5} \Psi_{2}-\eta_{2}^{*} B_{a b}^{*} \bar{\Psi}_{2} \Gamma^{a b} \gamma^{5} \Psi_{1}\right)
\end{aligned}
$$

describe how the two types of fermions couple to the background scalar field $\Phi$ and the two form field $B_{a b}$, respectively. The details of the boundary term and the definitions of gamma 
matrices in the bulk are shown in the appendix D. In the absence of the interaction terms, there are two free Dirac fermions in the bulk leading to two independent sets of chiral fermions in the field theory and therefore the interactions are necessary. These interaction terms take similar forms as that in the field theoretical model, although there are several other possible interaction terms. For example, one may also couple the two fermions to $B_{a b}$ with no insertion of $\gamma^{5}$. The reason for this choice is to produce the nodal band structure and corresponding topological properties of the nodal line semimetal $[8,9]$.

The equations of motion for these two fermions are

$$
\begin{gathered}
\left(\Gamma^{a} D_{a}-m_{f}\right) \Psi_{1}-\eta_{1} \Phi \Psi_{2}+\eta_{2} B_{a b} \Gamma^{a b} \gamma^{5} \Psi_{2}=0, \\
\left(\Gamma^{a} D_{a}+m_{f}\right) \Psi_{2}-\eta_{1}^{*} \Phi^{*} \Psi_{1}-\eta_{2}^{*} B_{a b}^{*} \Gamma^{a b} \gamma^{5} \Psi_{1}=0 .
\end{gathered}
$$

After taking the ansatz

$$
\Psi_{l}=\left(-g g^{r r}\right)^{-1 / 4} \psi_{l} e^{-i \omega t+i k_{x} x+i k_{y} y+i k_{z} z}=(u f)^{-1 / 2} \psi_{l} e^{-i \omega t+i k_{x} x+i k_{y} y+i k_{z} z},
$$

where $l=1,2$ and $\psi_{l}=\psi_{l}(r)$ are only functions of radial coordinate, (3.4) can be simplified as

$$
\begin{aligned}
\left(\Gamma^{\underline{r}} \partial_{r}+\frac{1}{u}\left(-i \omega \Gamma^{\underline{t}}+i k_{z} \Gamma^{\underline{z}}\right)+\frac{1}{\sqrt{u f}}\left(i k_{x} \Gamma^{\underline{x}}+i k_{y} \Gamma^{\underline{y}}\right)+(-1)^{l} \frac{m_{f}}{\sqrt{u}}\right) \psi_{l} \\
-\left(\eta_{1} \frac{\phi}{\sqrt{u}}+2 \eta_{2}\left((-1)^{l} \frac{\mathcal{B}_{x y}}{\sqrt{u} f} \Gamma^{x y}-i \frac{\mathcal{B}_{t z}}{\sqrt{u} u} \Gamma^{\underline{t z}}\right) \gamma^{5}\right) \psi_{3-l}=0 .
\end{aligned}
$$

In this equation, the couplings in the second line mixed the two different fermions and we have assumed that the couplings $\eta_{1}$ and $\eta_{2}$ are real numbers. Note that comparing with $[8,9]$, the last term in (3.6) is new. However, this new term does not change the asymptotic behaviors of this equation both in the IR and UV limits.

In the following, we introduce the necessary steps in calculating the fermionic spectral function including the boundary conditions in both IR and UV regime [8, 9, 33]. We also define the topological Hamiltonian and its related eigenstates in terms of the fermionic spectral function from holography $[8,9]$. To keep the main text straightforward, we take the NLSM phase as the example and leave details in other phases in appendix D. The system (3.6) contains eight first order, non-homogeneous differential equations and we shall solve them numerically with proper boundary conditions. We start from the asymptotic IR region $(r \rightarrow 0)$, where the nonlinear (coupling) terms become irrelevant and the equations can be analytically solved to leading order. For example in the nodal line semimetal phase with $\omega$ or $k_{z}$ finite, when $r \rightarrow 0$,

$$
\frac{1}{u} \propto \frac{1}{r^{2}}, \quad \frac{1}{\sqrt{u f}} \propto \frac{1}{r^{1+\alpha / 2}}, \quad \frac{\mathcal{B}_{x y}}{\sqrt{u} f} \propto \frac{\mathcal{B}_{t z}}{\sqrt{u} u} \propto \frac{1}{r} .
$$

Since $\psi_{1}, \psi_{2}$ are in the same order of $r$, then the equations can be simplified into decoupled forms describing two independent free fermions

$$
\left(\Gamma^{\underline{r}} \partial_{r}+\frac{1}{u_{0} r^{2}}\left(-i \omega \Gamma^{\underline{t}}+i k_{z} \Gamma^{\underline{z}}\right)\right) \psi_{l}=0 .
$$


Using the 2-components spinors $\psi_{l \pm}$ defined in appendix D, the first-order spinor differential equations can be simplified to second order scalar differential equations

$$
\frac{u_{0}^{2} r^{4}}{\omega^{2}-k_{z}^{2}} \partial_{r}^{2} \psi_{l+}+\frac{2 u_{0}^{2} r^{3}}{\omega^{2}-k_{z}^{2}} \partial_{r} \psi_{l+}+\psi_{l+}=0
$$

while $\psi_{l-}$ is determined by $\psi_{l+}$. After taking the explicit representation of the gamma matrices introduced in the appendix D, one can obtain the leading order infalling solutions analytically expressed as

$$
\psi_{l}^{\mathrm{IR}} \simeq e^{i \frac{\sqrt{\omega^{2}-k_{z}^{2}}}{u_{0} r}}\left(\begin{array}{c}
z_{1}^{l}(1+\ldots) \\
z_{2}^{l}(1+\ldots) \\
i \frac{\sqrt{\omega^{2}-k_{z}^{2}}}{\omega-k_{z}} z_{1}^{l}(1+\ldots) \\
i \frac{\sqrt{\omega^{2}-k_{z}^{2}}}{\omega+k_{z}} z_{2}^{l}(1+\ldots)
\end{array}\right)
$$

where $z_{1}^{l}, z_{2}^{l}$ are free constants, and the dots represent the higher order corrections with respect to $r$. Note that these IR behavior is the same as the one in $[8,9]$ and this is due to that the scaling symmetry of the IR geometry in NLSM here is the same as $[8,9]$.

Close to the UV boundary, similar to the case in the IR region, several terms become irrelevant and the equations take a simple form

$$
\left(r \Gamma^{\underline{r}} \partial_{r}-m_{f}\right) \psi_{1}=0, \quad\left(r \Gamma^{\underline{r}} \partial_{r}+m_{f}\right) \psi_{2}=0 .
$$

The masses determine the conformal dimensions of the fermionic operators, while our choice of coupling forms $S_{\Phi}$ and $S_{B}$ do not change the conformal dimensions. In components the Dirac fields can be solved as

$$
\psi_{1}=\left(\begin{array}{c}
s_{1} r^{m_{f}}+\ldots \\
s_{2} r^{m_{f}}+\ldots \\
r_{3} r^{-m_{f}}+\ldots \\
r_{4} r^{-m_{f}}+\ldots
\end{array}\right), \quad \psi_{2}=\left(\begin{array}{c}
r_{1} r^{-m_{f}}+\ldots \\
r_{2} r^{-m_{f}}+\ldots \\
s_{3} r^{m_{f}}+\ldots \\
s_{4} r^{m_{f}}+\ldots
\end{array}\right)
$$

where the coefficients $s_{i}$ and $r_{i}$ depend on the choices of $z_{1}^{l}$ and $z_{2}^{l}$ and can be solved numerically.

In this paper we will focus on the case with $m_{f}=-\frac{1}{4} \cdot{ }^{8}$ We take alternative quantization for $\psi_{1}$ while standard quantization for $\psi_{2}$, i.e., in both cases the dominant modes that proportional to $r^{-m_{f}}$ as the operator while the subdominant modes proportional to $r^{m_{f}}$ as the external source. The source and the response are

$$
\psi_{s}=\left(\begin{array}{c}
s_{1} \\
s_{2} \\
s_{3} \\
s_{4}
\end{array}\right), \quad \psi_{r}=\left(\begin{array}{c}
-r_{1} \\
-r_{2} \\
r_{3} \\
r_{4}
\end{array}\right) .
$$

\footnotetext{
${ }^{8}$ We expect our results are independent of the choice of $m_{f}$ since the near horizon boundary condition does not depend on the mass parameter. Nevertheless, it is still interesting to perform explicit numerical study to investigate whether richer effects might appear if we vary $m_{f}$.
} 
With the infalling boundary conditions (3.10) in IR, (3.6) can be solved numerically and we can get $\psi_{s}$ and $\psi_{r}$. The spinor operator $\psi_{r}$ and the spinor source $\psi_{s}$ are related by $\psi_{r}(k)=-i \Xi(k) \psi_{s}(k)$ where $\Xi(k)$ is a $4 \times 4$ matrix. To compute the matrix $\Xi(k)$, we need at least four sets of linearly independent sources and repsonses which can be obtained with four independent infalling boundary conditions at IR. We have a matrix equation for $\Xi(k)$

$$
M_{r}=-i \Xi M_{s},
$$

where

$$
M_{r}=\left(\psi_{r}^{I}, \psi_{r}^{I I}, \psi_{r}^{I I I}, \psi_{r}^{I V}\right), \quad M_{s}=\left(\psi_{s}^{I}, \psi_{s}^{I I}, \psi_{s}^{I I I}, \psi_{s}^{I V}\right)
$$

are $4 \times 4$ matrices. By right multiplying $M_{s}^{-1}$ on both sides of (3.14), one obtains $\Xi=$ $i M_{r} M_{s}^{-1}$, leading to the retarded Green's function for the fermionic operator

$$
G_{R}(k)=i \Gamma^{\underline{t}} M_{r}(k) M_{s}^{-1}(k) .
$$

The effective Hamiltonian is defined as

$$
H_{\mathrm{eff}}(\vec{k}) \equiv-G_{R}^{-1}(0, \vec{k})
$$

which is a generalization of topological Hamiltonian that is first introduced as a probe to detect topological invariants in topological insulators [37, 38]. We will study the topological invariants from this topological Hamiltonian in section 4.

\subsection{Numerical results}

In the previous subsection, we have introduced how to calculate the fermionic spectral function from holography. In the following, we explain our numerical results. Similar to the case without self-duality in $[8,9]$, we find that there exist multiple Fermi surfaces in the holographic nodal line semimetal phase and the dispersion close to the Fermi surface is linear. This observation is obtained from numerical calculation of the eigenvalues of the effective Hamiltonian (3.17) from which the band structure at $k_{z}=0$ plane in the momentum space is studied.

More precisely, since we have assumed the existence of $\mathrm{SO}(2)$-symmetry in the $x-y$ plane, we fix $k_{y}=0$ and compute $H_{\text {eff }}\left(k_{x}, k_{y}=k_{z}=0\right)$ without loss of generality and the locations of $H_{\text {eff }}=0$ indicates the nodal circle of Fermi surfaces. The four eigenvalues appear in two pairs and can be arranged as $\left\{h_{1},-h_{1}, h_{2},-h_{2}\right\}$ with $h_{1}, h_{2} \geq 0$ varies as a function of $k_{x}$. We show the effective band structure in the nodal line semimetal phase for $M / b \simeq 0.0014$ in figure 4 , where the red and blue curves are used to represent two different groups of bands. For convenience, we use "Band-1" and "Band-2" to describe the bands in red and blue curves in the following. The most interesting observation from this effective band structure is the existence of multiple and discrete Fermi surfaces ${ }^{9}$ for both energy bands indicated by band crossing at $h_{1}=h_{2}=0$. These Fermi surfaces appear alternately in "Band-1" and "Band-2", and more densely as the momentum $k_{x}$ decrease. We have checked that this characteristic band structure generically exist in the nodal

\footnotetext{
${ }^{9}$ Multiple Fermi surfaces in holography has also been found in [39-41] for a finite density system.
} 

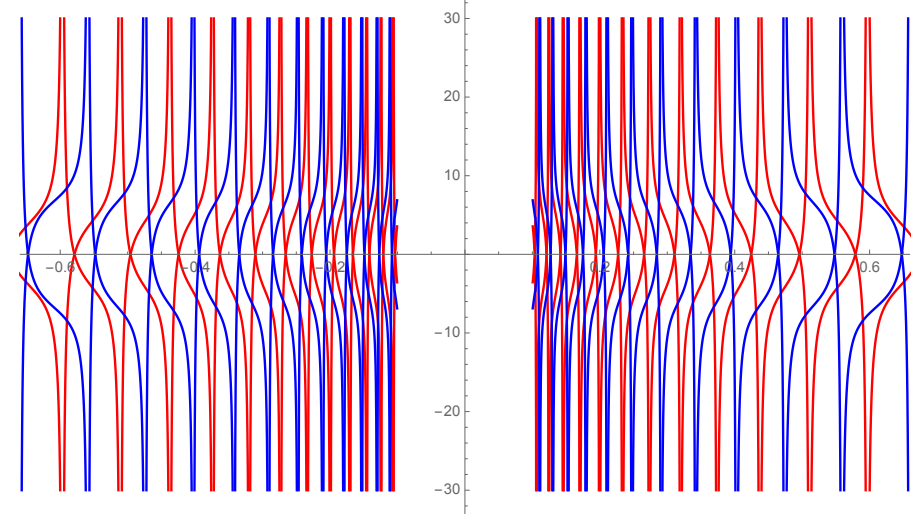

Figure 4. Eigenvalues of $G^{-1}\left(0, k_{x}, k_{y}=k_{z}=0\right)$ in the holographic NLSM phase as a function of $k_{x}$ for $M / b \simeq 0.0014$, in which blue and red curves represent two different sets of eigenvalues separately. Therefore one concludes that multiple Fermi surfaces exist in the NLSM phase.

line semimetal phase, not limited to particular values of $M / b$. Then a natural question arise that, are these band crossings accidental or topologically nontrivial? To answer this question, one should study the topological invariant and we leave to the next section.

The existence of nodal line shaped Fermi surface is the signature of NLSM. In the weakly coupled model, the Fermi surface appears at $k_{z}=0$, while for finite $k_{z}$ there exists only gapped band structure. Figure 4 shows that there exist multiple Fermi surfaces at $k_{z}=0$ in the strongly-coupled NLSM. Next we will explain that there is no Fermi surface for finite $k_{z}$ from the analysis of the fermionic spectral function. Note that the fermionic spectral function is defined from the imaginary part of the retarded Green's function $G_{R}(\omega, \vec{k})$, from which a Fermi surface can be identified when there is a sharp peak in $\operatorname{Im}\left[G_{R}(\omega \rightarrow 0, \vec{k})\right][31,32]$. The IR boundary condition for the fermions in the NLSM phase is shown is appendix D.4 and one concludes that

- for $k_{z}=0$ while $\omega \rightarrow 0$, the IR boundary condition is complex, which leads to the complex $G_{R}(\omega \rightarrow 0, \vec{k})$;

- for finite $k_{z}$ while $\omega \rightarrow 0$, the IR boundary condition is pure real and there is no imaginary part in $G_{R}(\omega \rightarrow 0, \vec{k})$.

Therefore, there is no Fermi surface when $k_{z} \neq 0$ since the spectral function vanishes. In addition, it is a special case when $\omega=0$ and we can identify the Fermi surface via the effective Hamiltonian (3.17) that gives consistent results as that from the spectral function.

In the vicinity of the Fermi surface, the dispersion relation of the excitations is linear in $k_{x}$. We make a generalization for the effective Hamiltonian (3.17) to finite $\omega \ll 1$ case. From the locations of zeros in $H_{\text {eff }}$ the dispersion relation can be obtained. As shown in figure 5 , we choose several discrete frequencies, get the locations of zeros and then plot these points in the dimensionless $\omega-k_{x}$ plane. We use power law functions to fit the data and find that close to the Fermi surface in the small $\omega$ region, the almost linear function perfectly fit 


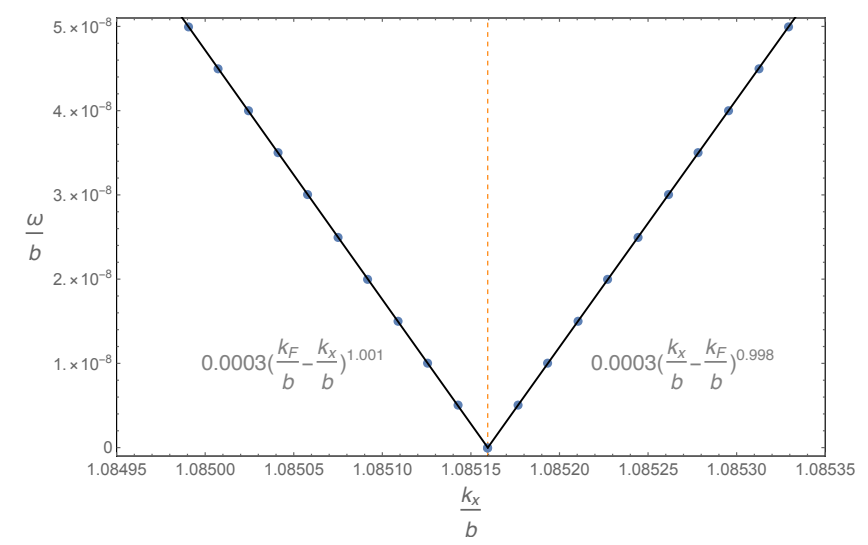

Figure 5. The linear dispersion around one of the multiple Fermi surfaces at $k_{F} / b=1.085$ for $\frac{M}{b} \simeq 0.0014$.

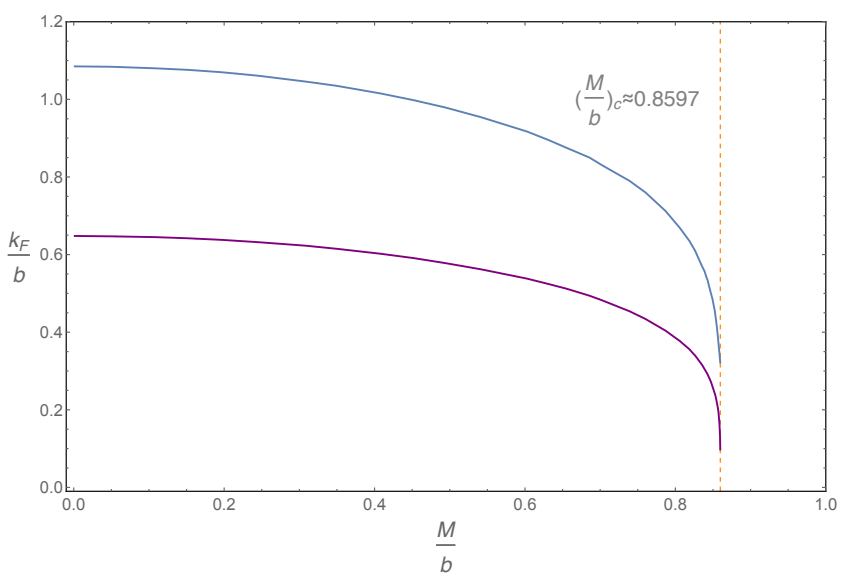

Figure 6. The size of fermi surfaces shrinks as $M / b$ increases. Fermi surfaces can exist in the nodal line semimetal phase as well as at the critical point while disappear in the trivial phase. The sudden disappearance of Fermi surfaces can be viewed as the consequence of the holographic quantum phase transition.

the data. The linear dispersion property of the excitations around a circular Fermi surface is another evidence to indicate that the ground state is a nodal line semimetal.

The quantum phase transition from the topological nodal line semimetal phase to a trivial phase can also be reflected from the effective band structure. This can be observed that the size of nodal circles $k_{F}=\sqrt{k_{x}^{2}+k_{y}^{2}}$ shrink as $M / b$ increases. ${ }^{10}$ We demonstrate the shrinking of two different Fermi surfaces in figure 6 where $k_{F} / b$ is a smooth, monotonic decreasing function when $0<M / b<0.8597$, which is a generic feature for all the Fermi surfaces and indicates that multiple Fermi surfaces cannot be removed from small perturbations of the ground state. In the topological trivial phase, there is no Fermi surface other than $k_{F}=0$ since from the near horizon we know that the retarded fermionic

\footnotetext{
${ }^{10}$ An interesting observation is that, the size of Fermi surfaces shrink in the holographic superfluids when the temperature is increased, e.g. in $[34,35]$. In the zero temperature topological NLSM phase, the size of Fermi surfaces shrinks as $M / b$ changes instead of temperature.
} 
Green's function is real for all values of spacelike $k^{\mu}[8,9,33]$. Finally, $k_{F}$ does not vanish at the critical point, which is different from the weakly-coupled theory. The reason is that, even though the form of interactions in (3.3) is a general approach to realize the nodal circle band structure, the couplings also deform the shape of the spectra. Therefore, it is reasonably expected that by fine-tuning $\eta_{1}$ and $\eta_{2}$, the Fermi surface could exactly shrink to zero at the critical point.

\section{Topological invariant in nodal line semimetal}

In the previous section, we have studied the effective band structure of the strongly coupled nodal line semimetal from the topological Hamiltonian of the fermionic Green's function in holography. The results, especially the existence of multiple nodal lines and linear dispersion close to each nodal line, show many similarities to the case without self-duality [8, 9]. In this section we will further study the topological property of these nodal lines.

We will study the topological invariants in the holographic semimetal phase to tell whether the nodal lines can be removed by small perturbations. In the nodal line semimetal system, one of the topological invariants is Berry phase which is defined on a closed path enclosing a node along the line $[3,30]$. By "enclosing" we mean that the path do not touch the node and the node cannot get out of the closed loop without cutting down the loop. The nodal line is accidental when the Berry phase associated with a closed loop enclosing a node on this line is 0 while topological protected if the Berry phase is $\pi$. In the following, we will discuss the Berry phase in both field theory and holography.

\subsection{Topological invariant in field theory}

We first calculate Berry phase from the weakly coupled field theory where the eigenvalues and eigenstates can be obtained analytically. Without loss of generality we set $b_{x y}=$ $1 / 2, m=0$ in the Lagrangian (2.5). With these parameters the system is in the nodal line semimetal phase and the nodal line is located on the $k_{x}-k_{y}$ plane with $k_{z}=0$ in the momentum space. The choice of the closed path along which we shall compute the Berrry phase is shown in figure 7. Note that we have the nodal line which is described by the red circle, and each point on this circle is a Weyl node. We focus on one single Weyl node and use a closed loop to enclose this point. For example, we choose $k_{y}=0$ and the closed path can be parametrized as

$$
\left(k_{x}, k_{z}\right)=\left(k_{F}+l \sin \theta, l \cos \theta\right), \quad \text { with } \theta \in[0,2 \pi) .
$$

Since we will generalize the discussion to strongly coupled theory in which the eigenstates can only be obtained at discrete points, we select a series of discrete points along the path, which is illustrated in the right plot in figure 7 . We have $\theta$ in (4.1) with $\theta_{i}=\frac{2 \pi i}{N}$ with $i \in\{1, \ldots, N\}$.

By making the circle discrete as above, we can define the discrete Berry phase

$$
e^{-i \phi_{i_{1} i_{2}}}=\frac{\left\langle n_{i_{1}} \mid n_{i_{2}}\right\rangle}{\left|\left\langle n_{i_{1}} \mid n_{i_{2}}\right\rangle\right|}
$$



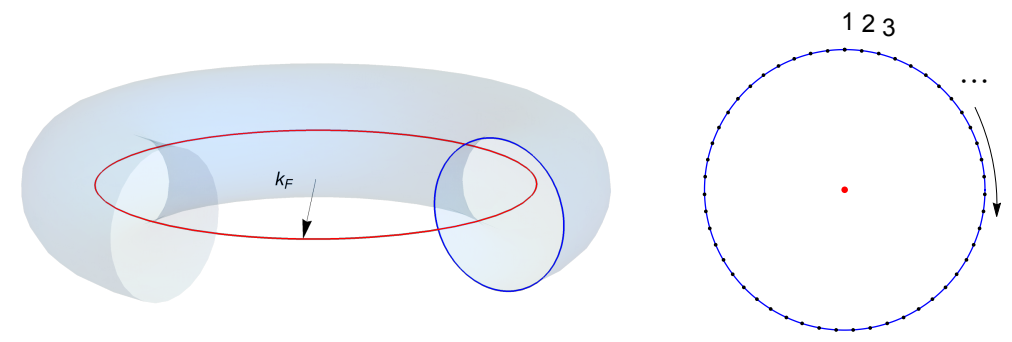

Figure 7. Left: the illustration of the closed path (blue curve) that encloses a Weyl node along the nodal line (red curve). Right: a series of discrete black points are selected along the closed path.

where $\left|n_{i_{1}}\right\rangle$ and $\left|n_{i_{2}}\right\rangle$ are eigenstates at two adjacent discrete points $i_{1}$ and $i_{2}$ along the path. The total Berry phase along the closed path is then the summation of all the discrete phases.

When $k_{y}=0$ from the Lagrangian (2.5) and the parameters we chose above, we have the eigenstates

$$
\begin{gathered}
\left|n^{I}\right\rangle=\left(1, \frac{k_{z}+\sqrt{\left(k_{x}-2\right)^{2}+k_{z}^{2}}}{2-k_{x}}, \frac{k_{z}+\sqrt{\left(k_{x}-2\right)^{2}+k_{z}^{2}}}{k_{x}-2}, 1\right)^{T}, \\
\left|n^{I I}\right\rangle=\left(1, \frac{k_{z}-\sqrt{\left(k_{x}-2\right)^{2}+k_{z}^{2}}}{2-k_{x}}, \frac{k_{z}-\sqrt{\left(k_{x}-2\right)^{2}+k_{z}^{2}}}{k_{x}-2}, 1\right)^{T},
\end{gathered}
$$

and the associated energy eigenvalues $E^{I}=-\sqrt{\left(k_{x}-2\right)^{2}+k_{z}^{2}}, \quad E^{I I}=\sqrt{\left(k_{x}-2\right)^{2}+k_{z}^{2}}$. For the choice of the "discrete" circle with (4.1), we have either states with energy $-l$ or $l$. By substituting (4.1) and (4.3) into (4.2) and do the summation of all the discrete phases, we find that the nontrivial phase factor is from the point where the normalized norm of two adjacent eigenstates becomes -1 . For states with energy $-l$, i.e. $\left|n^{I}\right\rangle$, due to $\left\langle n^{I}\left(k_{x}=2+0_{-}, k_{z} \simeq l\right) \mid n^{I}\left(k_{x}=2+0_{+}, k_{z} \simeq l\right)\right\rangle<0$ we have a phase factor $\pi$ from (4.2). Similarly for states with energy $l$, i.e. $\left|n^{I I}\right\rangle$, the phase factor $\pi$ of the Berry phase is due to $\left\langle n^{I I}\left(k_{x}=2+0_{-}, k_{z} \simeq-l\right) \mid n^{I I}\left(k_{x}=2+0_{+}, k_{z} \simeq-l\right)\right\rangle<0$. Therefore one concludes that Berry phase associated with a closed loop enclosing the node is $\pi$, which means that the nodal line semimetal described by (2.5) is topologically nontrivial.

\subsection{Topological invariant in holography}

In this subsection we compute Berry phase in the strongly coupled NLSM system from holography. Similar to the discussion in field theory, in holography we first compute the eigenstates of the effective Hamiltonian at a series of discrete points along a closed path in momentum space. The choice of discrete points and the definition of Berry phase are the same as the previous subsection for field theory, as shown in figure 7. Also, the Fermi surfaces only locate at the $k_{z}=0$ plane in the momentum space since the imaginary part of $G_{R}(\omega \rightarrow 0, \vec{k})$ vanishes for finite $k_{z}$, as explained in 3.2 in detail. The difference comparing to the field model is that, now there are multiple and dense nodal lines in the effective band structure. We should be careful that the closed loop encloses only a single Weyl node.

Note that the IR boundary conditions become a little bit tricky in this case because $k_{x}$ and $k_{z}$ are finite simultaneously while the $\mathrm{SO}(2)$ symmetry in the $k_{x^{-}} k_{z}$ plane is broken at 

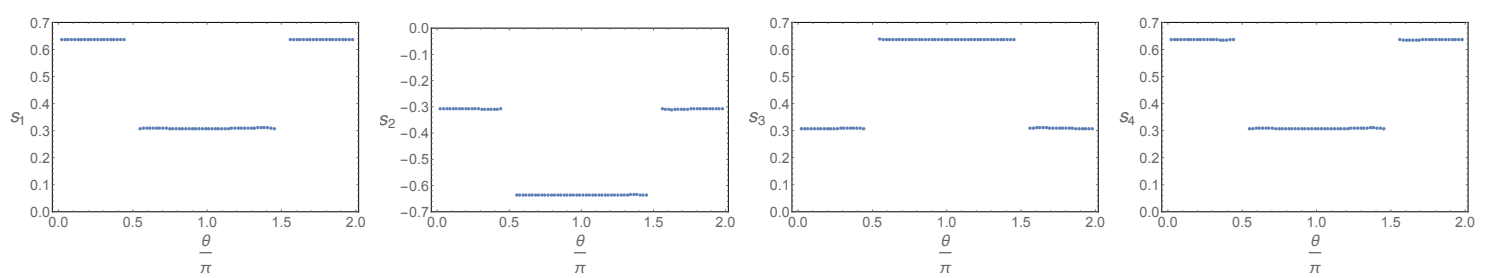

Figure 8. The eigenstates $\left(s_{1}, s_{2}, s_{3}, s_{4}\right)^{T}$ of $G^{-1}(0, \vec{k})$ as a function of $\theta / \pi$ along the enclosed loop with negative energy eigenvalues. Note that momentum is parameterized in (4.1). The sudden jump appears in all the four components of eigenstates $s_{i}$ across the $k_{x}$-axis i.e. at $\theta=\frac{1}{2} \pi, \frac{3}{2} \pi$.

low energy. When $k_{z}$ is finite, for example in the same order compared to $k_{x}$, the $k_{x}$-term in (3.6) can always be ignored since $r^{-1-\alpha / 2} \ll r^{-2}$. Instead, for the discrete points close to the $k_{x}$-axis where $k_{z}$ is very small, in the deep IR the $k_{x}$-term in (3.6) can not ignored any more and will modify the IR boundary conditions for the bulk fermions. However, whenever $k_{z}$ is nonzero one can always choose a sufficiently small $r$ such that the $k_{z}$ term dominates in (3.6). Therefore one could expect that the modification from finite $k_{x}$ on the ingoing spinor wave will not change the Berry phase. We list the IR boundary conditions for the cases with nonzero $k_{z}$ and $k_{z}=0$ in appendix D. In the following we first show the numerical results of the eigenstates and eigenvalues along the "discrete" circle.

We numerically show the dependence of the value of four components in the eigenstates on momentum for the negative energy eigenvalues in figure 8. As shown in this figure, each component poses sudden jump when across the $k_{x}$-axis from the upper plane to lower or vice versa. This is a common numerical result which does not depend on the radius of the "discrete" circle. Since the eigenstates change discontinuously across the $k_{x}$-axis, we should be careful to deal with points along the $k_{x}$-axis, i.e., to analyze the eigenstates for $k_{z}=0$ cases.

When there is no sudden jump in the eigenstates, the normalized norm of the adjacent states in (4.2) is one which contributes trivial to the phase factor of the Berry phase. The normalized norm at location of the sudden jump in the eigenstates is crucial for the phase factor which happens at $\theta=\pi / 2,3 \pi / 2$, similar to the case in the weakly coupled field theory. We label the states close to this momentum according to figure 9 .

Close to these points $\theta=\pi / 2,3 \pi / 2$, the eigenvalues and the corresponding eigenstates are summarized as follows in table 1 , where $b>a>0$. The values of $a, b$ are not important for analyzing the Berry phase. ${ }^{11}$ Substituting all these eigenstates into the discrete version of Berry phase, only the inner products formed by the eigenstates in this table give out non-vanishing phase. Similar to the case of field theory, for each band we can compute the Berry phase for states with either positive or negative energy of eigenvalues.

With the states in table 1 the calculation with respect to "Band-1" gives rise to a non-trivial phase $\pi$. However, the Berry phase for the nodal lines in "Band-2" cannot be determined due to that the norm of the adjacent states vanishes close to $k_{x}=0$.

\footnotetext{
${ }^{11}$ The eigenstates are also quantitively related to the couplings $\eta_{1}, \eta_{2}$, but qualitatively the same as figure 8 . Therefore, the Berry phase and further the existence of the topological invariant does not rely on the choice of couplings.
} 


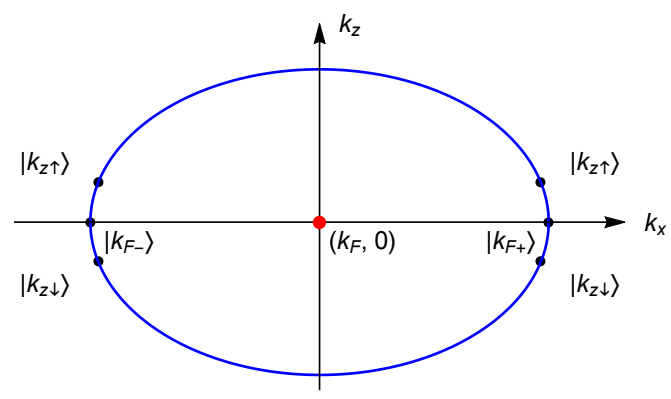

Figure 9. The diagram to illustrate the label of states which contributes nontrivially to the discrete Berry phase. The normalized inner product given by the adjacent points in the same upper or lower plane is one and gives a vanishing contribution to Berry phase. The net contribution of nontrivial discrete Berry phase is from the combination of $\left\langle k_{z \uparrow} \mid k_{F-}\right\rangle,\left\langle k_{F-} \mid k_{z \downarrow}\right\rangle,\left\langle k_{z \downarrow} \mid k_{F+}\right\rangle$ and $\left\langle k_{F+} \mid k_{z \uparrow}\right\rangle$, which can give a minus sign.

\begin{tabular}{|c|c|c|c|c|}
\hline & $\left|k_{z \uparrow}\right\rangle$ & $\left|k_{z \downarrow}\right\rangle$ & $\left|k_{F-}\right\rangle$ & $\left|k_{F+}\right\rangle$ \\
\hline$E_{1}<0$ & $(b,-a, a, b)^{T}$ & $(a,-b, b, a)^{T}$ & $\frac{1}{2}(1,-1,1,1)^{T}$ & $\frac{1}{2}(1,1,-1,1)^{T}$ \\
\hline$E_{1}>0$ & $(a, b,-b, a)^{T}$ & $(b, a,-a, b)^{T}$ & $\frac{1}{2}(1,1,-1,1)^{T}$ & $\frac{1}{2}(1,-1,1,1)^{T}$ \\
\hline$E_{2}<0$ & $(b,-a, a, b)^{T}$ & $(a,-b, b, a)^{T}$ & $\frac{1}{2}(1,1,1,-1)^{T}$ & $\frac{1}{2}(1,-1,-1,-1)^{T}$ \\
\hline$E_{2}>0$ & $(a, b,-b, a)^{T}$ & $(b, a,-a, b)^{T}$ & $\frac{1}{2}(1,-1,-1,-1)^{T}$ & $\frac{1}{2}(1,1,1,-1)^{T}$ \\
\hline
\end{tabular}

Table 1. We use $E_{1}$ and $E_{2}$ for eigenvalues of the states along discrete circles around "Band-1" and "Band-2". The states in the table are the ones which contribute to the nontrivial phase factor in the Berry phase. Note that here $b>a>0$.

All these features are qualitatively the same as those in the previous model $[8,9]$, indicating that the self-duality condition on the two form operators does not change the topological property in the strongly coupling regime. It seems that the topological property depends crucially on the action of the probe fermion while not the geometric background. ${ }^{12}$ This might indicate that there exist a semi-holographic description for the probe fermion similar to the case of probes with non-interacting Dirac equation [42], from which the topological properties might be clearly shown. It would be extremely interesting to construct this semi-holographic description.

\section{Conclusion and discussion}

We have considered an improved holographic nodal line semimetal model in which the duality relation between the rank two operators $\bar{\psi} \gamma^{\mu \nu} \psi$ and $\bar{\psi} \gamma^{\mu \nu} \gamma^{5} \psi$ in the dual field theory is satisfied. Following the approach in an AdS/QCD model [27], in holography we chose a special Chern-Simons term together with a mass term for the two form field

\footnotetext{
${ }^{12}$ This is related to the fact that the probe fermionic operators are $1 / N$ suppressed and their contributions to the geometric background is not visible at leading order.
} 
to realize the duality constraint automatically. In this improved holographic nodal line semimetal, we found that there still exists a quantum phase transition from topological nodal line semimetal phase to the topologically trivial phase.

We also calculated the fermionic spectral function by probing a massive Dirac fermion coupled in a particular way to the background field in bulk. We have found that, multiple nodal circle of Fermi surfaces exist in the topological NLSM phase while disappear in the trivial phase. The dispersion relation of low-energy excitations near the Fermi surfaces is linear. By tuning the dimensionless parameter $M / b$, the size of each nodal circle shrinks in the NLSM phase. These behaviors especially the discontinuity of Fermi circle in band structure indicate that a quantum phase transition happens at the critical point. We also computed the Berry phases in the NLSM phase and found that nodal circles are topologically nontrivial, which confirms the topological property of NLSM phase and the broken of topology across the quantum phase transition,

One might expect that after imposing the duality constraint new physics appears, while we have seen that most of the features above are qualitatively similar to the holographic model in $[8,9]$. The role of the duality in the two form operators does not modify the phase diagram qualitatively. The properties of the fermionic spectral function seems to depend crucially on the exact form the action of the probe fermions, while not on the geometric background. In condensed matter literature, the shear viscosity at low temperatures has been proposed in [43] as a probe to detect the band topology and the topological quantum phase transition. Therefore, it would be interesting to study other physical quantities, like transports [43-48] and non-local quantities to show some special features in this improved holographic model which serves as a natural ground to explore physics of strongly coupled NLSM.

\section{Acknowledgments}

We would like to thank Elias Kiritsis, Karl Landsteiner and Ya-Wen Sun and especially Carlos Hoyos for useful discussions. This work is supported in part by the National Natural Science Foundation of China grant No.11875083.

\section{A Equations of motion}

The equations of motion from the action (2.6) in the main text are

$$
\begin{aligned}
R_{a b}-\frac{1}{2} g_{a b}(R+12)-T_{a b} & =0, \\
\nabla_{b} \mathcal{F}^{b a}+2 \alpha \epsilon^{a b c d e} F_{b c} \mathcal{F}_{d e} & =0, \\
\nabla_{b} F^{b a}+\alpha \epsilon^{a b c d e}\left(F_{b c} F_{d e}+\mathcal{F}_{b c} \mathcal{F}_{d e}\right) & \\
-i q_{1}\left(\Phi^{*} D^{a} \Phi-\left(D^{a} \Phi\right)^{*} \Phi\right)+\frac{q_{2}}{\eta} \epsilon^{a b c d e} B_{b c} B_{d e}^{*} & =0, \\
D_{a} D^{a} \Phi-\partial_{\Phi^{*}} V_{1}-\lambda \Phi B_{a b}^{*} B^{a b} & =0, \\
\frac{i}{3 \eta} \epsilon_{a b c d e} H^{c d e}-m_{2}^{2} B_{a b}-\lambda \Phi^{*} \Phi B_{a b} & =0,
\end{aligned}
$$


where

$$
\begin{aligned}
T_{a b}= & \frac{1}{2}\left[\mathcal{F}_{a c} \mathcal{F}_{b}{ }^{c}-\frac{1}{4} g_{a b} \mathcal{F}^{2}\right]+\frac{1}{2}\left[F_{a c} F_{b}{ }^{c}-\frac{1}{4} g_{a b} F^{2}\right]+\frac{1}{2}\left(\left(D_{a} \Phi\right)^{*} D_{b} \Phi+\left(D_{b} \Phi\right)^{*} D_{a} \Phi\right)(\text { A.2 }) \\
& +\left(m_{2}^{2}+\lambda|\Phi|^{2}\right)\left(B_{a c}^{*} B_{b}{ }^{c}+B_{b c}^{*} B_{a}^{c}\right)-\frac{1}{2}\left(\left(D_{c} \Phi\right)^{*}\left(D^{c} \Phi\right)+V_{1}+V_{2}+\lambda|\Phi|^{2} B_{c d}^{*} B^{c d}\right) g_{a b}
\end{aligned}
$$

is the energy-momentum tensor. We make use of the following ansatz for the zerotemperature solutions,

$$
\begin{aligned}
d s^{2} & =u\left(-d t^{2}+d z^{2}\right)+\frac{d r^{2}}{u}+f\left(d x^{2}+d y^{2}\right), \\
\Phi & =\phi(r) \\
B_{x y} & =-B_{y x}=\mathcal{B}_{x y}, \\
B_{t z} & =-B_{z t}=i \mathcal{B}_{t z} .
\end{aligned}
$$

The equations of motion can be explicitly written as

$$
\begin{aligned}
\frac{u^{\prime \prime}}{u}+\frac{f^{\prime \prime}}{f}+\frac{1}{3}\left(\frac{u^{\prime 2}}{2 u^{2}}+\frac{7 u^{\prime} f^{\prime}}{2 u f}-\frac{f^{\prime 2}}{f^{2}}\right)-\frac{8}{u}+\frac{2}{3} \phi^{\prime 2}+\frac{2}{3 u}\left(m_{1}^{2} \phi^{2}+\frac{\lambda_{1}}{2} \phi^{4}\right) & =0 \\
\frac{u^{\prime \prime}}{u}-\frac{f^{\prime \prime}}{f}+\frac{u^{\prime}}{2 u}\left(\frac{u^{\prime}}{u}-\frac{f^{\prime}}{f}\right)-4\left(m_{2}^{2}+\lambda \phi^{2}\right)\left(\frac{\mathcal{B}_{t z}^{2}}{u^{3}}+\frac{\mathcal{B}_{x y}^{2}}{u f^{2}}\right) & =0 \\
\phi^{\prime \prime}+\left(\frac{3 u^{\prime}}{2 u}+\frac{f^{\prime}}{f}\right) \phi^{\prime}-\left(m_{1}^{2}+\lambda_{1} \phi^{2}-\frac{2 \lambda \mathcal{B}_{t z}^{2}}{u^{2}}+\frac{2 \lambda \mathcal{B}_{x y}^{2}}{f^{2}}\right) \frac{\phi}{u} & =0 \\
\mathcal{B}_{t z}^{\prime}-\frac{\eta \sqrt{u}}{2 f}\left(m_{2}^{2}+\lambda \phi^{2}\right) \mathcal{B}_{x y} & =0 \\
\mathcal{B}_{x y}^{\prime}-\frac{\eta f}{2 u^{\frac{3}{2}}}\left(m_{2}^{2}+\lambda \phi^{2}\right) \mathcal{B}_{t z} & =0
\end{aligned}
$$

There is an extra first order constraint equation which can be expressed as linear combinations of the previous equations and their derivatives

$$
\frac{\phi^{\prime 2}}{2}-\left(\frac{u^{\prime 2}}{4 u^{2}}+\frac{f^{\prime 2}}{4 f^{2}}+\frac{u^{\prime} f^{\prime}}{u f}\right)+\frac{6}{u}-\frac{1}{2 u}\left(m_{1}^{2} \phi^{2}+\frac{1}{2} \lambda_{1} \phi^{4}\right)+\left(\frac{m_{2}^{2}+\lambda \phi^{2}}{u}\right)\left(\frac{\mathcal{B}_{t z}^{2}}{u^{2}}-\frac{\mathcal{B}_{x y}^{2}}{f^{2}}\right)=0 .
$$

\section{B Counterterms and on-shell action}

To make the gravitational theory well behaved in variation and remove the divergence in the on-shell action, the Gibbons-Hawking term $S_{\mathrm{GH}}$ and the counterterms $S_{\text {c.t }}$ should be considered to construct the renormalized action

$$
S_{\text {ren }}=S+S_{\mathrm{GH}}+S_{\text {c.t }},
$$

where

$$
\begin{aligned}
S_{\mathrm{GH}} & =\int_{\partial} d^{d} x \sqrt{-h}(2 K) \\
S_{\mathrm{c.t}} & =\int_{\partial} d^{d} x \sqrt{-h}\left[-6-\Phi^{2}+\frac{1}{2}\left|B_{\mu \nu}\right|^{2}+\ln r\left(\left(\frac{1}{3}+\frac{\lambda_{1}}{2}\right) \Phi^{4}+\left|B_{\mu \nu}\right|^{4}\right)\right]
\end{aligned}
$$


are defined on the boundary of the bulk. $K=h^{a b} \nabla_{a} n_{b}$ is the trace of the extrinsic curvature of the induced metric $h_{a b}=g_{a b}-n_{a} n_{b}$ with $n_{a}=\left(0,0,0,0, \frac{1}{\sqrt{u}}\right)$ the spacelike normal vector, and $h$ is the determinant of the induced metric reduced onto the hypersurface orthogonal to $n_{a}$, i.e., $h \equiv \operatorname{det} h_{\mu \nu(\mu, \nu \neq r)}$.

Close to the AdS boundary $(r \rightarrow \infty)$, the expansions of the fields are

$$
\begin{aligned}
\left.u\right|_{r \rightarrow \infty} & =r^{2}-2 b^{2}-\frac{M^{2}}{3}+\left(\frac{4 b^{4}}{9}+\frac{23 M^{4}}{180}\right) \frac{\ln (r)}{r^{2}}+\frac{u_{2}}{r^{2}}+\ldots \\
\left.f\right|_{r \rightarrow \infty} & =r^{2}-\frac{M^{2}}{3}+\left(\frac{4 b^{4}}{9}+\frac{23 M^{4}}{180}\right) \frac{\ln (r)}{r^{2}}+\frac{f_{2}}{r^{2}}+\ldots \\
\left.\phi\right|_{r \rightarrow \infty} & =\frac{M}{r}-\frac{23 M^{3}}{60} \frac{\ln (r)}{r^{3}}+\frac{\phi_{2}}{r^{3}}+\ldots \\
\left.\mathcal{B}_{t z}\right|_{r \rightarrow \infty} & =b r-2 b^{3} \frac{\ln (r)}{r}+\frac{b_{t z 2}}{r}+\ldots \\
\left.\mathcal{B}_{x y}\right|_{r \rightarrow \infty} & =b r+2 b^{3} \frac{\ln (r)}{r}+\frac{b_{x y 2}}{r}+\ldots
\end{aligned}
$$

where

$$
\begin{aligned}
f_{2} & =\frac{7 b^{4}}{18}+\frac{b b_{x y 2}}{3}+\frac{5 b^{2} M^{2}}{18}+\frac{149 M^{4}}{1440}-\frac{u_{2}}{2}-\frac{M \phi_{2}}{2} \\
b_{t z 2} & =-b^{3}-b_{x y 2}-\frac{7 b M^{2}}{6}
\end{aligned}
$$

together with $\left\{b, M, u_{2}, b_{x y 2}, \phi_{2}\right\}$ are the coefficients of the series expansions. We have numerically checked that when $b=1$ is fixed, all the coefficients of the series expansions change smoothly by tuning $M$, even pass across the critical point. The free energy density is $\frac{\Omega}{V}=-\frac{S_{\text {o.s }}}{V}$ can be expressed by these coefficients

$$
S_{\text {o.s }}=\frac{1}{b^{4}}\left(\frac{11 b^{4}}{9}-\frac{8 b b_{x y 2}}{3}-\frac{38 b^{2} M^{2}}{9}-\frac{7 M^{4}}{36}+3 u_{2}+2 M \phi_{2}\right)
$$

which means that the free energy density is also smooth through the phase transition as illustrated in figure 3 in the main text.

\section{Scaling symmetries and numerical calculation}

The following scaling symmetries are very useful for numerical calculations.

- $\left\{r^{-1}, t, x, y, z\right\} \rightarrow\left\{\tilde{r}^{-1}, \tilde{t}, \tilde{x}, \tilde{y}, \tilde{z}\right\}=b\left\{r^{-1}, t, x, y, z\right\}$, while $\left\{u, f, B_{\mu \nu}\right\} \rightarrow$ $\left\{\tilde{u}, \tilde{f}, \tilde{B}_{\mu \nu}\right\}=b^{-2}\left\{u, f, B_{\mu \nu}\right\}$ to make $d s^{2}$ and $B=B_{\mu \nu} d x^{\mu} d x^{\nu}$ remain unchanged according to this transformation. This symmetry can be used to fix $b$ to be 1 .

- $\{x, y\} \rightarrow\{\tilde{x}, \tilde{y}\}=c\{x, y\}$ together with $\left\{f, B_{x y}\right\} \rightarrow\left\{\tilde{f}, \tilde{B}_{x y}\right\}=c^{-2}\left\{f, B_{x y}\right\}$ indicates another scaling symmetry that is restricted in the $x-y$ plane. This symmetry can scale $f$ to asymptotic to $r^{2}$ near the boundary. It also makes us possible to fix a shooting parameter in the IR region, since we can make a transformation back to the expected coordinates. 


\section{Dirac system in the bulk}

\section{D.1 Vielbein and spin connection}

The vielbein is a two indexed object with a tangent space index $\underline{m}$, and a coordinate index $a$. It obeys the relations $e_{\underline{m}}^{a} e_{\underline{n}}^{b} g_{a b}=\eta_{\underline{m n}}$ and $\eta_{\underline{m n}} e^{\frac{m}{a}} e_{b}^{n}=g_{a b}$. For a diagonal metric (2.9), we have

$$
e \frac{m}{a}=\sqrt{\left|g_{a a}\right|} \delta_{\frac{m}{a}}
$$

where $a$ does not sum. The tangent space index $\underline{m}$ is lowered or raised by Minkowski metric $\eta_{\underline{m n}}$ or $\eta^{\underline{m n}}$, while the coordinate index is lowered or raised by $g_{a b}$ or $g^{a b}$. The spin connection can be constructed from the vielbein and the Christoffel symbol

$$
\omega_{a}^{\underline{m}}{ }_{\underline{n}}=\Gamma_{a c}^{b} e_{\frac{m}{b}} e_{\underline{n}}^{c}-e_{\underline{n}}^{b} \partial_{a} e_{\frac{m}{b}} .
$$

The covariant derivative of the spinor can be defined as

$$
\nabla_{a}=\partial_{a}-\frac{i}{4} \omega_{a \underline{m n}} \Gamma \underline{m n} .
$$

\section{D.2 Gamma matrices and spinors}

We use the Pauli matrices

$$
\sigma_{x}=\left(\begin{array}{ll}
0 & 1 \\
1 & 0
\end{array}\right), \quad \sigma_{y}=\left(\begin{array}{cc}
0 & -i \\
i & 0
\end{array}\right), \quad \sigma_{z}=\left(\begin{array}{cc}
1 & 0 \\
0 & -1
\end{array}\right), \quad I_{2}=\left(\begin{array}{ll}
1 & 0 \\
0 & 1
\end{array}\right) .
$$

to build up the gamma-matrices $\gamma^{\mu}$ in the 4-dimensional flat space-time

$$
\gamma^{0}=\left(\begin{array}{cc}
0 & i I_{2} \\
i I_{2} & 0
\end{array}\right), \quad \gamma^{i}=\left(\begin{array}{cc}
0 & i \sigma_{i} \\
-i \sigma_{i} & 0
\end{array}\right), \quad \gamma^{5}=i \gamma^{0} \gamma^{1} \gamma^{2} \gamma^{3}=\left(\begin{array}{cc}
-I_{2} & 0 \\
0 & I_{2}
\end{array}\right) \text {. }
$$

The gamma matrices $\Gamma^{\underline{a}}$ in the 5 -dimensional local flat space-time can be constructed

$$
\Gamma^{\underline{a}}=\left(\Gamma^{\underline{\mu}}, \Gamma^{\underline{r}}\right) \equiv\left(\gamma^{\mu},-\gamma^{5}\right)
$$

Gamma matrices $\Gamma^{a}$ in the 5-dimensional curved space-time are

$$
\Gamma^{a}=e_{\underline{m}}^{a} \Gamma^{\underline{m}} .
$$

The Clifford algebras for the above cases are

$$
\left\{\gamma^{\mu}, \gamma^{\nu}\right\}=2 \eta^{\mu \nu}, \quad\left\{\Gamma^{\underline{a}}, \Gamma^{\underline{b}}\right\}=2 \eta^{\underline{a b}}, \quad\left\{\Gamma^{a}, \Gamma^{b}\right\}=2 g^{a b} .
$$

The two-indexed anti-symmetrized products of gamma matrices are defined as

$$
\gamma^{\mu \nu}=\frac{i}{2}\left[\gamma^{\mu}, \gamma^{\nu}\right], \quad \Gamma^{\underline{a b}}=\frac{i}{2}\left[\Gamma^{\underline{a}}, \Gamma^{\underline{b}}\right], \quad \Gamma^{a b}=\frac{i}{2}\left[\Gamma^{a}, \Gamma^{b}\right]=e_{\underline{m}}^{a} e_{\underline{n}}^{b} \Gamma^{\underline{m n}} .
$$

A spinor $\psi$ can be decomposed into the right-handed and left-handed spinors $\psi_{R, L}$ defined as

$$
\psi_{R}=\left(\begin{array}{c}
\psi_{+} \\
0
\end{array}\right), \quad \psi_{L}=\left(\begin{array}{c}
0 \\
\psi_{-}
\end{array}\right)
$$

with the projection operator constructed from $\Gamma^{\underline{r}}$

$$
\frac{1}{2}\left(1 \pm \Gamma^{\underline{r}}\right) \psi=\psi_{R, L}
$$

where $\psi_{ \pm}$are two-components spinors. 


\section{D.3 UV boundary terms of Dirac equations}

In this part, we briefly review how to obtain the correct boundary action for a single chiral fermion at the boundary $[33,49,50]$. Next, we review the generalization to combining two opposite chiral fermions into a massive Dirac fermion with correct boundary action.

We start from the action for a single, free Dirac fermion with mass $m$ in the bulk

$$
S_{\text {Dirac }}=\int d^{d+1} x \sqrt{-g}\left(\bar{\psi} \Gamma^{a} \nabla_{a} \psi-m \bar{\psi} \psi\right) .
$$

Note that the boundary is defined at $r \rightarrow \infty$. The variation of the action is

$$
\delta S_{\text {Dirac }}=\text { bulk term }+\int_{\partial \mathcal{M}} d^{d} x \sqrt{-g g^{r r}}\left(\bar{\psi}_{L} \delta \psi_{R}-\bar{\psi}_{R} \delta \psi_{L}\right)
$$

where the bulk terms give the dynamical equations of motion. The last terms are located at the AdS boundary where $\psi_{R}$ and $\psi_{L}$ as varied independently. However, because the bulk Dirac equation is of first order, $\psi_{R}$ and $\psi_{L}$ are related and only one of them can be varied freely while the other behaves as the corresponding response. This can be achieved by adding a proper boundary term to the original action [33, 49, 50].

For example, if we choose $\psi_{R}$ as free variable, we add

$$
S_{\partial}=\int_{\partial \mathcal{M}} d^{d} x \sqrt{-g g^{r r}} \bar{\psi}_{R} \psi_{L}
$$

and the variation of the boundary field theory is

$$
\delta S_{\text {total }}=\delta\left(S_{\text {bulk }}+S_{\partial}\right)=\int_{\partial \mathcal{M}} d^{d} x \sqrt{-g g^{r r}}\left(\bar{\psi}_{L} \delta \psi_{R}+\delta \bar{\psi}_{R} \psi_{L}\right) .
$$

Alternatively, we can take $\psi_{L}$ as the free variable by considering the boundary term

$$
S_{\partial}=\int_{\partial \mathcal{M}} d^{d} x \sqrt{-g g^{r r}}\left(-\bar{\psi}_{L} \psi_{R}\right),
$$

leading to the alternative total variation as

$$
\delta S_{\text {total }}=\delta\left(S_{\text {bulk }}+S_{\partial}\right)=-\int_{\partial \mathcal{M}} d^{d} x \sqrt{-g g^{r r}}\left(\delta \bar{\psi}_{L} \psi_{R}+\bar{\psi}_{R} \delta \psi_{L}\right) .
$$

For five dimensional bulk theory, we introduced two sets of coupled fermions $\psi^{(1)}, \psi^{(2)}$ in (3.1). The boundary terms we considered are a combination of (D.14) and (D.16). Performing the variation, we end up with

$$
S_{\mathrm{bdy}}=\int d^{d} x \sqrt{-h}\left(\bar{\psi}_{R}^{(1)} \psi_{L}^{(1)}-\bar{\psi}_{L}^{(2)} \psi_{R}^{(2)}\right)
$$

where the mass of $\psi^{(1)}$ and $\psi^{(2)}$ are $m$ and $-m$, respectively. One may also notice that there is an opposite sign comparing (D.15) and (D.17). This results in the additional minus sign in (3.13) when identifying the 4-component fermionic operator. 


\section{D.4 IR boundary conditions}

We outline the infalling boundary conditions according to different phases and choices of $k_{\mu}=\left(\omega, k_{x}, k_{y}, k_{z}\right)$ in a table.

\begin{tabular}{|c|c|c|}
\hline Phase & $k^{\mu}$ & IR infalling solution \\
\hline \multirow{2}{*}{ NLSM } & $\omega$ or $k_{z} \neq 0, \forall k_{x}, k_{y}$ & $\psi_{l}^{\mathrm{IR}}=e^{i \frac{\sqrt{\omega^{2}-k_{z}^{2}}}{u_{0}}}\left(z_{1}^{l}, z_{2}^{l}, i \frac{\sqrt{\omega^{2}-k_{z}^{2}}}{\omega-k_{z}} z_{1}^{l}, i \frac{\sqrt{\omega^{2}-k_{z}^{2}}}{\omega+k_{z}} z_{2}^{l}\right)^{T}$ \\
\cline { 2 - 4 } & $\omega=k_{z}=0, k_{x}$ or $k_{y} \neq 0$ & $\psi_{l}^{\mathrm{IR}}=e^{-\frac{2 \tilde{k}_{x}}{\alpha} r^{-\frac{\alpha}{2}}}\left(z_{1}^{l}, z_{2}^{l}, z_{2}^{l}, z_{1}^{l}\right)^{T}$ \\
\hline Critical & $\omega=k_{z}=0, k_{x}$ or $k_{y} \neq 0$ & $\psi_{l}^{\mathrm{IR}}=e^{-\frac{2 \tilde{k}_{x}}{\alpha_{c}} r^{-\frac{\alpha_{c}}{2}}}\left(z_{1}^{l}, z_{2}^{l}, z_{2}^{l}, z_{1}^{l}\right)^{T}$ \\
\hline Trivial & $\omega=k_{z}=0, k_{x}$ or $k_{y} \neq 0$ & $\psi_{l}^{\mathrm{IR}}=e^{-\frac{\tilde{k}_{x}}{r}}\left(z_{1}^{l}, z_{2}^{l}, z_{2}^{l}, z_{1}^{l}\right)^{T}$ \\
\hline
\end{tabular}

In this table $\tilde{k}_{x} \equiv \frac{k_{x}}{\sqrt{u_{0} f_{0}}}$ in NLSM phase, $\tilde{k}_{x} \equiv \frac{k_{x}}{\sqrt{u_{c} f_{c}}}$ at the critical point and $\tilde{k}_{x} \equiv \frac{k_{x}}{\sqrt{u_{1}}}$, with $\left(u_{0}, f_{0}, u_{c}, f_{c}, u_{1}\right)=\left(\frac{1}{8}(11+3 \sqrt{13}), \sqrt{\frac{2}{3} \sqrt{13}-2}, 2.735,0.754,1+\frac{3}{8 \lambda_{1}}\right)$.

Open Access. This article is distributed under the terms of the Creative Commons Attribution License (CC-BY 4.0), which permits any use, distribution and reproduction in any medium, provided the original author(s) and source are credited.

\section{References}

[1] A.A. Burkov, Weyl metals, Ann. Rev. Condensed Matter Phys. 9 (2018) 359 [arXiv: 1704.06660] [INSPIRE].

[2] N.P. Armitage, E.J. Mele and A. Vishwanath, Weyl and Dirac semimetals in three dimensional solids, Rev. Mod. Phys. 90 (2018) 015001 [arXiv:1705.01111] [InSPIRE].

[3] C. Fang, H. Weng, X. Dai and Z. Fang, Topological nodal line semimetals, Chin. Phys. B 25 (2016) 117106 [arXiv:1609.05414].

[4] J. Zaanen, Y. Liu, Y.-W. Sun and K. Schalm, Holographic duality in condensed matter physics, Cambridge University Press, Cambridge, U.K. (2015).

[5] M. Ammon and J. Erdmenger, Gauge/gravity duality: foundations and applications, Cambridge University Press, Cambridge, U.K. (2015).

[6] S.A. Hartnoll, A. Lucas and S. Sachdev, Holographic quantum matter, arXiv:1612.07324 [INSPIRE].

[7] K. Landsteiner and Y. Liu, The holographic Weyl semi-metal, Phys. Lett. B 753 (2016) 453 [arXiv: 1505. 04772] [INSPIRE].

[8] Y. Liu and Y.-W. Sun, Topological nodal line semimetals in holography, JHEP 12 (2018) 072 [arXiv: 1801.09357] [INSPIRE].

[9] Y. Liu and Y.-W. Sun, Topological invariants for holographic semimetals, JHEP 10 (2018) 189 [arXiv: 1809.00513] [INSPIRE].

[10] K. Landsteiner, Y. Liu and Y.-W. Sun, Quantum phase transition between a topological and a trivial semimetal from holography, Phys. Rev. Lett. 116 (2016) 081602 [arXiv:1511. 05505] [INSPIRE]. 
[11] K. Landsteiner, Y. Liu and Y.-W. Sun, Odd viscosity in the quantum critical region of a holographic Weyl semimetal, Phys. Rev. Lett. 117 (2016) 081604 [arXiv:1604.01346] [INSPIRE].

[12] C. Copetti, J. Fernández-Pendás and K. Landsteiner, Axial Hall effect and universality of holographic Weyl semi-metals, JHEP 02 (2017) 138 [arXiv:1611.08125] [INSPIRE].

[13] G. Grignani, A. Marini, F. Pena-Benitez and S. Speziali, AC conductivity for a holographic Weyl semimetal, JHEP 03 (2017) 125 [arXiv: 1612.00486] [INSPIRE].

[14] M. Ammon, M. Heinrich, A. Jiménez-Alba and S. Moeckel, Surface states in holographic Weyl semimetals, Phys. Rev. Lett. 118 (2017) 201601 [arXiv: 1612.00836] [INSPIRE].

[15] M. Ammon, M. Baggioli, A. Jiménez-Alba and S. Moeckel, A smeared quantum phase transition in disordered holography, JHEP 04 (2018) 068 [arXiv: 1802.08650] [INSPIRE].

[16] M. Baggioli, B. Padhi, P.W. Phillips and C. Setty, Conjecture on the butterfly velocity across a quantum phase transition, JHEP 07 (2018) 049 [arXiv: 1805.01470] [INSPIRE].

[17] Y. Liu and J. Zhao, Weyl semimetal/insulator transition from holography, JHEP 12 (2018) 124 [arXiv: 1809.08601] [INSPIRE].

[18] G. Song, J. Rong and S.-J. Sin, Stability of topology in interacting Weyl semi-metal and topological dipole in holography, JHEP 10 (2019) 109 [arXiv:1904.09349] [INSPIRE].

[19] X. Ji, Y. Liu and X.-M. Wu, Chiral vortical conductivity across a topological phase transition from holography, Phys. Rev. D 100 (2019) 126013 [arXiv:1904.08058] [INSPIRE].

[20] I. Tanaka, Holographic quantum singularity, arXiv:2001.06611 [INSPIRE].

[21] V. Juričić, I. Salazar Landea and R. Soto-Garrido, Phase transitions in a holographic multi-Weyl semimetal, JHEP 07 (2020) 052 [arXiv: 2005.10387] [INSPIRE].

[22] M. Baggioli and D. Giataganas, Detecting topological quantum phase transitions via the c-function, Phys. Rev. D 103 (2021) 026009 [arXiv:2007.07273] [InSPIRE].

[23] B. Kiczek, M. Rogatko and K.I. Wysokiński, Anomalous Hall conductivity of the holographic $Z_{2}$ Dirac semimetals, arXiv:2010.13095 [INSPIRE].

[24] U. Gürsoy, V. Jacobs, E. Plauschinn, H. Stoof and S. Vandoren, Holographic models for undoped Weyl semimetals, JHEP 04 (2013) 127 [arXiv:1209.2593] [INSPIRE].

[25] K. Bitaghsir Fadafan, A. O'Bannon, R. Rodgers and M. Russell, A Weyl semimetal from AdS/CFT with flavour, JHEP 04 (2021) 162 [arXiv: 2012.11434] [INSPIRE].

[26] K. Landsteiner, Y. Liu and Y.-W. Sun, Holographic topological semimetals, Sci. China Phys. Mech. Astron. 63 (2020) 250001 [arXiv:1911.07978] [INSPIRE].

[27] R. Alvares, C. Hoyos and A. Karch, An improved model of vector mesons in holographic QCD, Phys. Rev. D 84 (2011) 095020 [arXiv:1108.1191] [InSPIRE].

[28] A.A. Burkov, M.D. Hook and L. Balents, Topological nodal semimetals, Phys. Rev. B $\mathbf{8 4}$ (2011) 235126 [arXiv:1110.1089].

[29] S.K. Domokos, J.A. Harvey and A.B. Royston, Completing the framework of AdS/QCD: $h_{1} / b_{1}$ mesons and excited wrho's, JHEP 05 (2011) 107 [arXiv:1101.3315] [INSPIRE].

[30] M.V. Berry, Quantal phase factors accompanying adiabatic changes, Proc. Roy. Soc. Lond. A 392 (1984) 45.

[31] M. Cubrovic, J. Zaanen and K. Schalm, String theory, quantum phase transitions and the emergent Fermi-liquid, Science 325 (2009) 439 [arXiv:0904.1993] [InSPIRE]. 
[32] H. Liu, J. McGreevy and D. Vegh, Non-Fermi liquids from holography, Phys. Rev. D 83 (2011) 065029 [arXiv:0903.2477] [INSPIRE].

[33] N. Iqbal and H. Liu, Real-time response in AdS/CFT with application to spinors, Fortsch. Phys. 57 (2009) 367 [arXiv:0903.2596] [InSPIRE].

[34] S.S. Gubser, F.D. Rocha and A. Yarom, Fermion correlators in non-Abelian holographic superconductors, JHEP 11 (2010) 085 [arXiv: 1002.4416] [INSPIRE].

[35] M. Ammon, J. Erdmenger, M. Kaminski and A. O'Bannon, Fermionic operator mixing in holographic p-wave superfluids, JHEP 05 (2010) 053 [arXiv: 1003.1134] [INSPIRE].

[36] N.W.M. Plantz, F. García Flórez and H.T.C. Stoof, Massive Dirac fermions from holography, JHEP 04 (2018) 123 [arXiv:1802.04191] [INSPIRE].

[37] Z. Wang and S.-C. Zhang, Simplified topological invariants for interacting insulators, Phys. Rev. X 2 (2012) 031008 [arXiv:1203.1028].

[38] Z. Wang and B. Yan, Topological Hamiltonian as an exact tool for topological invariants, J. Phys. Condens. Matter 25 (2013) 155601 [arXiv:1207.7341] [INSPIRE].

[39] S.A. Hartnoll, D.M. Hofman and D. Vegh, Stellar spectroscopy: fermions and holographic Lifshitz criticality, JHEP 08 (2011) 096 [arXiv:1105.3197] [INSPIRE].

[40] N. Iqbal, H. Liu and M. Mezei, Semi-local quantum liquids, JHEP 04 (2012) 086 [arXiv: 1105.4621] [INSPIRE].

[41] M. Cubrovic, Y. Liu, K. Schalm, Y.-W. Sun and J. Zaanen, Spectral probes of the holographic Fermi groundstate: dialing between the electron star and AdS Dirac hair, Phys. Rev. D 84 (2011) 086002 [arXiv: 1106.1798] [INSPIRE].

[42] T. Faulkner and J. Polchinski, Semi-holographic Fermi liquids, JHEP 06 (2011) 012 [arXiv: 1001.5049] [INSPIRE].

[43] M. Moore, P. Surówka, V. Juričić and B. Roy, Shear viscosity as a probe of nodal topology, Phys. Rev. B 101 (2020) 161111 [arXiv:1912.07611] [InSPIRE].

[44] K. Landsteiner, Notes on anomaly induced transport, Acta Phys. Polon. B 47 (2016) 2617 [arXiv: 1610.04413] [INSPIRE].

[45] M. Ammon, S. Grieninger, A. Jimenez-Alba, R.P. Macedo and L. Melgar, Holographic quenches and anomalous transport, JHEP 09 (2016) 131 [arXiv:1607.06817] [INSPIRE].

[46] Y. Bu, T. Demircik and M. Lublinsky, Nonlinear chiral transport from holography, JHEP 01 (2019) 078 [arXiv: 1807.08467] [INSPIRE].

[47] M. Haack, D. Sarkar and A. Yarom, Probing anomalous driving, JHEP 04 (2019) 034 [arXiv: 1812.08210] [INSPIRE].

[48] M. Ammon et al., Chiral hydrodynamics in strong external magnetic fields, JHEP 04 (2021) 078 [arXiv: 2012.09183] [INSPIRE].

[49] M. Henneaux, Boundary terms in the AdS/CFT correspondence for spinor fields, in International meeting on mathematical methods in modern theoretical physics (ISPM 98), (1998) [hep-th/9902137] [INSPIRE].

[50] J.N. Laia and D. Tong, A holographic flat band, JHEP 11 (2011) 125 [arXiv:1108.1381] [INSPIRE]. 